\title{
SHOCK WAVES AND CERENKOV RADIATION IN ELECTROMAGNETIC SUBSTRATUM
}

\author{
H.E. WILHELM \\ Department of Materials Science \& Engineering \\ University of Utah, Salt Lake City, Utah 84110, U.S.A. \\ (Received April 4, 1991 and in revised form March 6, 1991)
}

\begin{abstract}
The EM fields of charged particles moving with velocity $\mathbf{v}$ in a physical vacuum with wave carrier (substratum) are determined by means of the generalized, Galilei covariant Maxwell equations for inertial frames $\Sigma$ with substratum flow w. In this Galilean approach, all velocities have absolute meaning relative to the substratum rest frame $\Sigma^{\circ}$, and the relative velocity of material particles is given by the linear Galilean relation $\mathbf{v}_{G}=v_{1}-v_{2}$, permitting in principle superluminal relative velocities $\left|v_{G}\right|>c_{0}$. Inter alias, the possibility of EM shock waves and Cerenkov radiation in the vacuum substratum is discussed. Experiments are proposed to test the theoretical predictions.
\end{abstract}

KEY WORDS AND PHRASES. Electromagnetic theory, Cerenkov radiation, vacuum substratum, Galilei covariance. 1980 MATHEMATICS SUBJECT CLASSIFICATION CODE. 78A02; 78A25; 78A40.

\section{INTRODUCTION.}

The excitation of electromagnetic (EM) shock waves by a charged particle moving in vacuum with a velocity $v>c_{o}$ larger than the velocity of light $c_{o}$ in free space was suggested in 1904 by Sommerfeld. [1] In the course of the subsequent development of the special theory of relativity, this idea was not further investigated, since the STR does not permit particle velocities $|\mathbf{v}| \geq c_{o}$ relative to an accelerator. [2] On the experimental side, in a dielectric with refractive index $n(\omega)$ $>1$ and phase velocity of light $\mathrm{c}=\mathrm{c}_{\mathrm{o}} / \mathrm{n}<\mathrm{c}_{\mathrm{o}}$, EM shock waves have been generated by means of electron beams with subluminal relativistic velocities $c<|v|<c_{0}$. [3] The emitted EM waves are in the visible region, and were discovered by Cerenkov. [3]

In this connection, it should be understood that the relative velocity of two systems with velocities $v_{1}$ and $v_{2}$ is, in the STR, given by the nonlinear theorem $v_{R}=\left(v_{1}-v_{2}\right) /\left(1-v_{1} \bullet v_{2} / c_{o}^{2}\right)$ for parallel velocities, whereas the Galilean relative velocity, $v_{G}=v_{1}-v_{2}$, is linear. [2] E.g., if $v_{1,2}= \pm$ $0.9 c_{o}$, then $v_{R}=1.8 c_{o} / 1.81<c_{o}$ whereas $v_{G}=1.8 c_{o}>c_{o}$. For $v_{1,2}= \pm 10 c_{o}$, one would have $v_{R}=$ $20 c_{o} / 101 \approx c_{o} / 5$ whereas $v_{G}=20 c_{o}$. These examples are one of the many reasons for the widespread distrust in the physical meaningfulness of the STR. [2,4-18,20,35] As explained by Janossy, the nonlinear relative velocity $\mathbf{v}_{R}$ of the STR is an arbitrary ad-hoc definition, and its 
alleged agreement with experiments is due to the direct use of the STR in the interpretation of the experimental data. [2] He proposes to define the relative velocity of two systems in accordance with the Galilean relative velocity $\mathbf{v}_{\mathrm{G}}$, not only for low but in particular for high velocities $\mathbf{v}_{1,2}$, in the same representation. [2]

Within the frame of the STR, which implies that EM waves propagate in an empty vacuum without wave carrier (substructure or ether) [2], a Cerenkov effect in vacuum would be unthinkable since all electron beams are subluminal, $|\mathbf{v}|<c_{o}$, by definition. The experimentally unproven assertion of the STR that EM waves propagate in a vacuum free from any material wave carrier has come under increased criticism as a physical untenable concept (see, e.g., Ives [4,5], Janossy [2,6,7], Dirac [8,9], Builder [10,11], Mansouri and Sex] [12], or Winterberg [13-18]). Contrary to widespread misunderstandings, Einstein clearly recognized the physical necessity of a carrier medium for EM waves in vacuum in 1921. [19]

Even so we do not yet have a satisfactory microscopic theory of the EM wave carrier [2,420], it is remarkable that the STR (which denies the EM ether) "unintentionally" reintroduces an ether through the Lorentz covariant (i) quantum mechanics (Dirac's electron-positron vacuum) $[2,8,9,13,16,18]$ and (ii) quantum electrodynamics (EM zero-point energy). $[9,13,16,18]$ This ether of relativistic quantum mechanics and electrodynamics has infinite energy and mass densities, owing to the Lorentz covariance imposed on these theories. [13-18] As expected, these divergencies do not occur in Galilei covariant quantum mechanics and electrodynamics, which lead to a minimum length $r_{o}$ (non-point particles) in physics, and a maximum frequency $\omega_{0}=2 \pi c_{o} / r_{o}$ (finite zero-point energy density) for EM waves in vacuum. $[13,16,18]$ Experimentally, the existence of a preferred frame of reference (ether rest frame) is supported through the $2.7^{\circ} \mathrm{K}$ microwave background of the universe. [21,22]

In a physical vacuum with an EM substratum or wave carrier, EM shock waves appear to be producible even by means of subluminal electrons, $\mathbf{v}<c_{0}$, if the Galilean electron velocity $\mathbf{v}$ w relative to the EM wave carrier, which streams with the velocity $w$, can be larger than velocity of light $c_{o}$ in vacuum. The condition for the observation of the vacuum Cerenkov effect, $|\mathbf{v}-\mathbf{w}|$ $>c_{o}$, could then be realizable on the earth by pointing the electron beam into the direction antiparallel to the terrestrial ether flow $\mathbf{w}$, e.g. when $|\mathbf{v}-\mathbf{w}|=|\mathbf{v}|+|\mathbf{w}|>\mathrm{c}_{\mathrm{o}}$ even if $|\mathbf{v}|<\mathrm{c}_{\mathrm{o}}$. Measurements of the cosmic microwave background indicate terrestrial ether velocities of the order $w \sim 3 \times 10^{5} \mathrm{~m} / \mathrm{s}$. [21,22] Accordingly, on the earth the excitation of Cerenkov radiation in vacuum would require an electron beam moving with a velocity $v>c_{o}-3 \times 10^{5}[\mathrm{~m} / \mathrm{s}]$ upstream the terrestrial ether flow w.

In order to explain the mechanism of EM shock waves in vacuum, consider the familiar acoustic shock waves excited by a projectile moving with a supersonic speed, $v>c_{s}$, in air at rest $(\mathbf{w}=\mathbf{0})$. Schlieren photographs show that the acoustic shock wave has a wave front of the form of a Mach cone with vertex at the instantaneous position of the projectile. [23] Such an acoustic shock wave is also produced by a projectile moving with a subsonic velocity $v<c_{s}$ upstream a subsonic gas flow ( $w<c_{s}$ ) of a wind tunnel, when the (Galilean) velocity $\mathbf{v}-\mathbf{w}$ of the projectile relative to the gas flow is supersonic, $|\mathbf{v}-\mathbf{w}|>c_{s}$. [23] These experiments are in complete agreement with the theory of acoustic shock waves excited by a moving projectile in homogeneous 
gas flows. [23] Similarly, EM shock waves should be excited in vacuum by an electron beam with a Galilean velocity $|\mathbf{v}-\mathbf{w}|>c_{0}$ relative to the ether. Experimental verification of the EM shock waves in vacuum could be accomplished through observation of the emitted Cerenkov radiation.

The following considerations on the possibility of EM shock waves and Cerenkov radiation in a physical vacuum with a wave carrier (ether) are based on the generalized Galilei covariant Maxwell equations for inertial frames $\Sigma$ with ether flow w. [24-27] In this Galilei covariant theory, all velocities have an absolute meaning relative to the preferred ether rest frame $\Sigma^{\circ}$. The relative velocity of two systems with velocities $v_{1}$ and $v_{2}$ are given by the linear Galilean relation $v_{G}=v_{1}$ $v_{2}$, all velocities referring to the same inertial frame. In Galilean physics, relative velocities $v_{G}>$ $c_{o}$ are realizable by first principles, in agreement with experiments. [2] In view of the theoretical findings, experiments are proposed to test the principles of the Einsteinian and Galilean relativity concepts at high energies.

\section{THEORETICAL PRINCIPLES}

The carrier of the EM fields in vacuum has the electric permittivity $\epsilon_{\mathrm{o}} \approx 10^{-9} / 36 \pi[\mathrm{As} / \mathrm{Vm}]$ and the magnetic permeability $\mu_{0} \approx 4 \pi \times 10^{-9}$ [Vs/Am]. [2] The inertial frame, in which experiments show isotropic propagation of (one-way) light signals in vacuum with the wave speed $c_{\mathrm{o}}=\left(\mu_{\mathrm{o}} \epsilon_{\mathrm{o}}\right)^{-1 / 2} \approx 3 \times 10^{8}[\mathrm{~m} / \mathrm{s}]$, is defined as the rest frame $\Sigma^{\mathrm{o}}\left(\mathbf{r}^{\mathrm{o}}, \mathrm{t}^{\mathrm{o}}, \mathbf{0}\right)$ of the EM wave carrier $\left(\mathbf{w}^{\mathrm{o}}\right.$ $=0) \cdot[2,4-8,10-19,28]$ In this so-called EM substratum or ether frame, the ordinary Maxwell equations hold since $w^{0}=0$ in $\Sigma^{0}$. [2] Transforming the Maxwell equations for the ether rest frame $\Sigma^{\circ}\left(\mathbf{r}^{\mathrm{o}}, \mathbf{t}^{\mathrm{o}}, \mathbf{0}\right)$ to an arbitrary inertial frame $\Sigma(\mathbf{r}, \mathrm{t}, \mathbf{w})$ with ether flow $\mathbf{w}$, by means of the Galilean spacetime transformations, leads to generalized covariant Maxwell equations which contain explicitly the ether velocity $w$. [24-27] In particular, the wave equations for the magnetic vector potential $\mathbf{A}(\mathbf{r}, \mathbf{t})$ and the scalar electric potential $\Phi(\mathbf{r}, \mathbf{t})$ in vacuum $\left(\mu_{\mathrm{o}}, \epsilon_{\mathrm{o}}\right)$ are obtained for an arbitrary inertial frame $\Sigma(\mathbf{r}, \mathbf{t}, \mathbf{w})$ with ether flow $\mathbf{w}$ in the form (Chapter 6): [24-27]

$$
\begin{gathered}
{\left[\mu_{\mathrm{o}} \epsilon_{\mathrm{o}}(\partial / \partial \mathrm{t}+\mathbf{w} \bullet \nabla)^{2}-\nabla^{2}\right] \mathbf{A}=\mu_{\mathrm{o}}(\mathbf{j}-\rho \mathbf{w}),} \\
{\left[\mu_{\mathrm{o}} \epsilon_{\mathrm{o}}(\partial / \partial \mathrm{t}+\mathbf{w} \bullet \nabla)^{2}-\nabla^{2}\right](\Phi-\mathbf{w} \bullet \mathbf{A})=\rho / \epsilon_{\mathrm{o}},}
\end{gathered}
$$

where the divergence of $\mathbf{A}(\mathbf{r}, \mathbf{t})$ is determined by the generalized Lorentz gauge [24-27]

$$
\nabla \bullet \mathbf{A}=-\mu_{\mathrm{o}} \epsilon_{\mathrm{o}}(\partial / \partial \mathrm{t}+\mathbf{w} \bullet \nabla)(\Phi-\mathbf{w} \bullet \mathbf{A}) .
$$

The current density $j(r, t)$ and space charge density $\rho(r, t)$ fields are the sources of the EM potentials. From the solutions $\mathbf{A}(\mathbf{r}, \mathbf{t})$ and $\Phi(\mathbf{r}, \mathbf{t})$ of Eqs. (1) - (2), the EM vacuum fields $\mathbf{E}(\mathbf{r}, \mathbf{t})$ and $\mathbf{H}(\mathbf{r}, \mathbf{t})$ result as

$$
\mathbf{E}=-\nabla \Phi-\partial \mathbf{A} / \partial \mathbf{t}, \quad \mathbf{H}=\nabla \times \mathbf{A} / \mu_{\mathrm{o}} .
$$

Equations (1) - (4) are Galilei covariant, i.e., are of the same form in all inertial frames, since the operators $(\partial / \partial \mathrm{t}+w \bullet \nabla)=\left(\partial / \partial^{\prime}+w^{\prime} \bullet \nabla^{\prime}\right)$ and $\nabla=\nabla^{\prime}$ and the fields $\mathbf{A}(\mathbf{r}, \mathrm{t})=\mathbf{A}^{\prime}\left(\mathbf{r}^{\prime}, \mathrm{t}^{\prime}\right)$ and $\Phi(r, t)-\mathbf{w} \bullet A(r, t)=\Phi^{\prime}\left(\mathbf{r}^{\prime}, t^{\prime}\right)-w^{\prime} \bullet A^{\prime}\left(\mathbf{r}^{\prime}, t^{\prime}\right)$ are Galilei invariants (Chapter 6). [24-27]

A charge e with velocity $v(t)$ and instantaneous position $s(t)=r_{o}+\int_{0}^{t} v\left(t^{\prime}\right) d t^{\prime}$ at time $t$, has the current and space charge density fields, 


$$
\mathbf{j}(\mathbf{r}, \mathrm{t})=\rho(\mathbf{r}, \mathrm{t}) \mathbf{v}(\mathrm{t}), \quad \rho(\mathbf{r}, \mathrm{t})=\mathrm{e} \delta\left(\mathbf{r}-\mathbf{r}_{\mathrm{o}}-\int_{0}^{\mathrm{t}} \mathbf{v}(\tau) \mathrm{d} \tau\right) .
$$

In this case, $\mathbf{j}-\rho \mathbf{w}=\rho(\mathbf{v}-\mathbf{w})$, and Eq. (1) can be used to eliminate $\mathbf{A}(\mathbf{r}, \mathbf{t})$ from Eq. (2). Accordingly, the independent wave equation for the scalar potential of the moving charge is

$$
\left[\mu_{\mathrm{o}} \epsilon_{\mathrm{o}}(\partial / \partial \mathrm{t}+\mathbf{w} \bullet \nabla)^{2}-\nabla^{2}\right] \Phi=\left[1+\mathbf{w} \bullet(\mathbf{v}-\mathbf{w}) / \mathrm{c}_{\mathrm{o}}^{2}\right] \rho / \epsilon_{\mathrm{o}} .
$$

As a brief illustration to the above wave equations, consider the propagation of plane EM waves $\mathbf{A}(\mathbf{r}, \mathbf{t})=\mathbf{A}_{\omega} \exp (\mathrm{i} \omega \mathrm{t}-\mathrm{ik} \bullet \mathbf{r}$ ) with frequency $\boldsymbol{\omega}$ and wave length $\lambda=2 \pi / \mathbf{k}$ in an inertial frame $\Sigma(\mathbf{r}, t, \mathbf{w})$ with ether flow $\mathbf{w}$. With $\mathbf{j} \equiv \mathbf{0}, \rho \equiv 0$ (vacuum), Eq. (1) gives for these waves the dispersion

$$
\omega=k_{\mathrm{o}}+\mathbf{k} \bullet \mathbf{w} \text {. }
$$

Accordingly, EM waves propagating (i) downstream the ether flow (k॰w >0) and (ii) upstream the

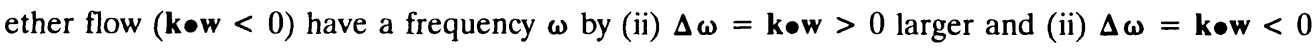
smaller, than the corresponding EM wave $\omega=k c_{0}$ in the ether rest frame $\Sigma^{\circ}\left(w^{0} \equiv 0\right)$. For terrestrial conditions, these effects are insignificant since $\omega=\mathrm{kc}_{\mathrm{o}}\left(1+\mathrm{k} \bullet \mathbf{w} / \mathrm{kc}_{\mathrm{o}}\right) \approx \mathrm{kc}_{\mathrm{o}}$ for $\mathrm{w} / \mathrm{c}_{\mathrm{o}} \sim$ $10^{-3}$. [21] Moreover, for the usual go $(+\mathbf{k})$ and return $(-\mathbf{k})$ propagation experiments, the net convective frequency shift vanishes, $\Delta \omega=\mathbf{w} \bullet(+\mathbf{k})+\mathbf{w} \bullet(-\mathbf{k})=0$.

Distant galaxies move away from the earth with relativistic velocities $0.1 c_{o}<v_{G}<0.9 c_{0}$. [29] It is conceivable that the EM ether moves along with the galaxies due to gravitation, $\mathbf{w}=\mathbf{v}_{\mathrm{G}}$. In this case, the convective ether effects would be significant since $w / c_{o}=v_{G} / c_{o} \leq 1$. By Eq. (7), EM waves propagating from a distant galaxy towards the earth $\left(k \bullet v_{G}<0\right)$ should exhibit a convective ether redshift $\Delta \boldsymbol{\omega}=\mathbf{k} \bullet \mathbf{w}=\mathbf{k} \bullet \mathbf{v}_{\mathrm{G}}<0$, which is of the magnitude of the observed anomalous redshift. [29]

In the theory of the ordinary Cerenkov effect for dielectrics with permittivity $\epsilon(\omega)>1$, the energy density of the emitted radiation is finite, since the necessary condition $\epsilon(\omega)>1$ is not satisfied for frequencies $\omega$ above an upper limit $\omega_{m}<\infty$. [30] Radiation theory in vacuum (frequency independent $\epsilon_{\mathrm{o}}$ and $\mu_{\mathrm{o}}$ ) leads, within the frame of the STR, to divergent results in the limit $\omega \rightarrow \infty$ or $k=2 \pi / \lambda \rightarrow \infty$. [30,31,32] As an illustration, we discuss briefly the infinite EM energy density of the vacuum predicted by Lorentz covariant quantum electrodynamics. $[30,13,16,18]$

EM field fluctuations persist in vacuum even at $\mathrm{T}=0^{\circ} \mathrm{K}$, in accordance with the uncertainty principle, $p_{\lambda} q_{\lambda}=\hbar / 2$ for all oscillator occupation numbers $N_{\lambda}=0$. The energy of the EM zeropoint fluctuations in a volume $\mathrm{V}$ of the vacuum is given by the least oscillator energy eigenvalue, $\Sigma_{\lambda} \hbar \omega_{\lambda}\left(N_{\lambda}+1 / 2\right)=\Sigma \hbar \omega_{\lambda} / 2$ for $N_{\lambda}=0$,

$$
\mathrm{W}_{\mathrm{o}}=\int_{\mathrm{v}^{\frac{1}{2}}}\left[\epsilon_{\mathrm{o}} \mathrm{E}(\mathbf{r})^{2}+\mu_{\mathrm{o}} \mathrm{H}(\mathbf{r})^{2}\right] \mathrm{d}^{3} \mathbf{r}=2 \Sigma_{\lambda} \frac{1}{2} \hbar \omega_{\lambda}
$$

where the factor "2" considers the polarizations of the transverse fields with eigenfrequency $\omega_{\lambda}$. For a sufficiently large volume $V$ the $\Sigma_{\lambda}$ is replaced by an integration over quasi-continuous modes with mode density $4 \pi \omega^{2} \mathrm{~d} \omega\left(4 \pi \mathrm{c}_{\mathrm{o}}\right)^{-3}$. Thus, one finds for the total EM energy density of the vacuum

$$
\mathrm{W}_{\mathrm{o}} \mathrm{N}=4 \pi \hbar\left(4 \pi \mathrm{c}_{\mathrm{o}}\right)^{-3} \int_{0}^{\infty} \omega^{3} \mathrm{~d} \omega=\infty .
$$

The spectral energy density $d W_{\alpha} / d \omega \propto \omega^{3}$, with $0 \leq \omega \leq \infty$, is the sole Lorentz covariant spectrum possible, and this causes the divergence in Eq. (9). The infinite energy density, $W_{\alpha} / V=\infty$, and mass density, $\mathrm{W}_{o} / \mathrm{Vc}_{\mathrm{o}}^{2}=\infty$, of the vacuum are physically untenable. This divergence is artificially 
produced by the Lorentz covariance imposed on the spectral EM energy density. $[14,16,18]$

Lorentz covariant quantum mechanics and electrodynamics predict unrealistic (unobservable) effects, such as infinite EM zero-point energy of vacuum, infinite self-energy of point-particles, selfacceleration of point-particles to the velocity of light, and numerous relativistic "paradoxes." [2,611,13-18,30-32] On the other hand, Galilei covariant physics does not require particles to be mathematical points, nor the existence of EM waves with infinite wave number $k=2 \pi / \lambda_{0}=\infty$ or frequency $\omega=2 \pi c_{o} / \lambda_{o}=\infty$. Actual particles $\left(r_{o}>0\right)$ can not be points $\left(r_{o}=0\right)$ and physical processes $\left(t_{o}>0\right)$ can not be instantaneous $\left(t_{o}=0\right)$. Following earlier proposals of Heisenberg [33] and Wheeler [34] for a minimum length $r_{o}>0$ and minimum time $t_{o}>0$ in physics, we restrict the wave numbers $k=2 \pi / \lambda$ and frequencies $\omega=k c_{o}$ of vacuum (ether rest frame) to non-infinite values,

$$
0 \leq \mathrm{k}<\mathrm{k}_{\mathrm{o}} \sim 1 / \mathrm{r}_{\mathrm{o}}, \quad 0 \leq \omega \leq \omega_{\mathrm{o}} \sim \mathrm{c}_{\mathrm{o}} / \mathrm{r}_{\mathrm{o}}
$$

where $r_{o}=c_{o} t_{0}$. As to theoretical attempts to determine $r_{o}$ and $t_{o}$, see Heisenberg, Wheeler, or Winterberg. $[33,34,13,14,16,18]$ In the case of the Cerenkov effect, we will see that a natural frequency cutoff results from the non-zero radius of the charged particle, which is large compared with the estimated minimum length in physics. [33,34]

\section{ELECTRODYNAMICS IN VACUUM}

EM signals advance in vacuum with a velocity which is independent of the velocity of the emitting source. $[2,6,7]$ Thus, the vacuum behaves like a carrier medium (ether) for EM waves, in a similar way as gases, liquids, or solids serve as carrier media for ordinary waves. Galilei covariant EM theory shows that EM signals propagate in an inertial frame $\Sigma(r, t, w)$ with the velocity $c_{o}+w$ downstream the ether flow $w$ and the velocity $c_{o}-w$ upstream the ether flow $w$. $[2,6,7,10,11,25,36]$ This interesting ether effect has been observed in Sagnac type experiments, but is unobservable in the usual Michelson-Morley type [2] setups using go-and-return light paths, due to the length contraction of the mirror distances from the semi-transparent plate. For this reason, the STR, which asserts that one and the same light signal propagates not only in the inertial frame of source but also in all other inertial frames with the same velocity $c_{o}$ ("relativity principle"), represents a tautology based not on actual light velocities but on average go-and-return light velocities $\bar{c}=c_{o}$. $[2,6,7,10,11,25,35]$

On the other hand, a particle moves in vacuum with a momentum mv, which depends not only on the imparted acceleration but also on the state of motion of the accelerator in the inertial frame $\Sigma(\mathbf{r}, \mathbf{t}, \mathbf{w})$ in which $\mathbf{v}$ is observed. Since no specific tests were made, experiments are not known which indicate that an accelerator produced (under the same operating conditions) a larger particle velocity $\mathbf{v}$ in a given direction $(v \bullet w>0)$ than in the opposite direction $(\mathbf{v} \bullet w<0)$. Since a particle does not propagate in the ether like an EM wave, there are no apriory reasons why a particle should not be accelerable to Galilean velocities $|\mathbf{v}-\mathbf{w}|>c_{0}$ relative to the ether in an inertial frame $\Sigma(\mathbf{r}, \mathbf{t}, \mathbf{w})$. However, if experiments are interpreted by means of the STR, relativistic particle velocities are always "relative" and subluminal, $\mathrm{v}_{\mathrm{R}}<\mathrm{c}_{\mathrm{o}}$ (relativistic addition theorem). E.g., if two systems move with velocities $v_{1,2}= \pm \alpha c_{0}$ in opposite directions, their relative velocity is $v_{R}$ 
$=\left(v_{1}-v_{2}\right) /\left(1-v_{1} v_{2} / c_{o}^{2}\right)=2 \alpha c_{d} d\left(1+\alpha^{2}\right)<c_{o}$ (subluminal) for any $\alpha>0$ according to the STR, whereas their Galilean relative velocity is $v_{G}=v_{1}-v_{2}=2 \alpha c_{o}>c_{o}$ (superluminal) for $\alpha>1 / 2$.

In order to investigate whether superluminal Galilean particle velocities $|\mathbf{v}-\mathbf{w}|>c_{0}$ relative to the streaming (w) ether are compatible with Galilei covariant physics, the EM field of a charged particle moving with arbitrary uniform velocity $\mathbf{v}$ in an inertial frame $\Sigma(r, t, w)$ is determined by means of the Galilei covariant EM field equations. [25] The implication is that velocities $\mathbf{v}$ of charged particles, for which the associated EM field is imaginary, are physically not realizable.

Consider a point charge e moving with a uniform velocity $\mathbf{v} \neq \mathbf{w}$ (in general) in an inertial frame $\Sigma(\mathbf{r}, \mathbf{t}, \mathbf{w})$ with ether flow w, FIG. 1. According to Eqs. (1) and (2), the EM vacuum potentials $A(r, t)$ and $\Phi(r, t)$ of this moving charge satisfy the wave equations of $\Sigma(r, t, w)$ :

$$
\begin{gathered}
{\left[\mu_{\mathrm{o}} \epsilon_{\mathrm{o}}(\partial / \partial \mathrm{t}+\mathrm{w} \bullet \nabla)^{2}-\nabla^{2}\right] \mathbf{A}=\mu_{\mathrm{o}}(\mathbf{j}-\rho \mathbf{w}),} \\
{\left[\mu_{\mathrm{o}} \epsilon_{\mathrm{o}}(\partial / \partial \mathrm{t}+\mathrm{w} \bullet \nabla)^{2}-\nabla^{2}\right](\Phi-w \bullet \mathbf{A})=\rho / \epsilon_{\mathrm{o}},}
\end{gathered}
$$

where

$$
\rho=\mathrm{e} \delta(\mathbf{r}-\mathrm{vt}), \quad \mathbf{j}=\mathrm{e} \delta(\mathbf{r}-\mathrm{vt}) \mathrm{v}
$$

are the charge and current densities of the particle. By Eqs. (11) - (13), A(r,t) is parallel to ( $\mathbf{v}-\mathbf{w})$. For this reason, a coordinate system $(x, y, z)$ is chosen with its $z$-axis in the direction of $(v-w)$, in which (FIG. 1)

$$
\mathbf{v}-\mathbf{w}=(\mathbf{v}-\mathrm{w})_{\mathrm{z}} \mathbf{a}_{\mathrm{z}}, \quad A=A(x, y, z, t) \mathbf{a}_{z} .
$$

In this coordinate system, Eqs. (11) - (13) reduce to the wave equations (see also Eq. (6)),

Hence,

$$
\begin{gathered}
{\left[\mu_{\mathrm{o}} \epsilon_{\mathrm{o}}(\partial / \partial \mathrm{t}+\mathrm{w} \bullet \nabla)^{2}-\nabla^{2}\right] \mathbf{A}(\mathrm{x}, \mathrm{y}, \mathrm{z}, \mathrm{t})=\mu_{\mathrm{o}} \mathrm{e} \delta\left(\mathrm{x}-\mathrm{v}_{\mathrm{x}} \mathrm{t}\right) \delta\left(\mathrm{y}-\mathrm{v}_{\mathrm{y}} \mathrm{t}\right) \delta\left(\mathrm{z}-\mathrm{v}_{\mathrm{z}} \mathrm{t}\right)(\mathrm{v}-\mathrm{w}),} \\
{\left[\mu_{\mathrm{o}} \epsilon_{\mathrm{o}}(\partial / \partial \mathrm{t}+\mathrm{w} \bullet \nabla)^{2}-\nabla^{2}\right] \Phi(\mathrm{x}, \mathrm{y}, \mathrm{z}, \mathrm{t})=\left(\mathrm{e} / \epsilon_{\mathrm{o}}\right)\left[1+\mathrm{w} \bullet(\mathrm{v}-\mathrm{w}) / \mathrm{c}_{\mathrm{o}}^{2}\right] \delta\left(\mathrm{x}-\mathrm{v}_{\mathrm{x}} \mathrm{t}\right) \delta\left(\mathrm{y}-\mathrm{v}_{\mathrm{y}} \mathrm{t}\right) \delta\left(\mathrm{z}-\mathrm{v}_{\mathrm{z}} \mathrm{t}\right) .}
\end{gathered}
$$

$$
A(r, t)=\mu_{o} \epsilon_{o}(v-w) \Phi(r, t) /\left[1+w \bullet(v-w) / c_{o}^{2}\right]
$$

Note that $r-v t=\left\{x-v_{x} t, y-v_{y} t, z-v_{z} t\right\}$ and $v-w=(v-w)_{z} a_{z}$, i.e., $v_{x}=w_{x}, v_{y}=w_{y}$, and $v_{z}=w_{z}+(v-w)_{z}$ $\equiv v_{z}$ in the chosen $x y z$ - system of coordinates. Eq. (17) permits finding $\Phi(r, t)$ from the solution for $A(r, t)$, and vice versa.

Since $\partial / \partial \mathrm{t}+\mathrm{v} \bullet \nabla=0$ for uniform charge motion $\mathbf{v}$, the mixed convective derivatives in Eqs. (15) and (16) can be eliminated by means of the operator identity.

$$
\partial / \partial \mathrm{t}+\mathrm{w} \bullet \nabla=\partial / \partial \mathrm{t}+\mathrm{v} \bullet \nabla \cdot(\mathbf{v}-\mathbf{w}) \bullet \nabla=-(\mathrm{v}-\mathrm{w})_{z} \partial / \partial_{z} .
$$

Thus Eq. (16) is reduced to a Poisson equation of the form

$$
\left(\partial^{2} / \partial x^{2}+\partial^{2} / \partial y^{2}+\partial^{2} / \partial \zeta^{2}\right) A(x, y, \zeta, t)=-\mu_{o} e(v-w) \delta\left(x-v_{x} t\right) \delta\left(y-v_{y} t\right) \delta\left(\sqrt{1-(v-w)^{2} / c_{o}^{2}} \zeta-v_{z} t\right)
$$

where

$$
\zeta=z /\left[1-(v-w)^{2} / c_{o}^{2}\right]^{1 / 2}
$$

is a dilated axial coordinate. Since the Green's function of Eq. (19) is $G\left(x, y, \zeta ; x^{\prime}, y^{\prime}, \zeta^{\prime}\right)=1 / 4 \pi[(x-$ $\left.\left.x^{\prime}\right)^{2}+\left(y-y^{\prime}\right)^{2}+\left(\zeta-\zeta^{\prime}\right)^{2}\right]^{1 / 2}$, the vector potential has the integral representation 


$$
\begin{aligned}
& A(x, y, \zeta, t)=\left(\mu_{0} e / 4 \pi\right)(v-w) \iiint_{-\infty}^{+\infty}\left[\left(x-x^{\prime}\right)^{2}+\left(y-y^{\prime}\right)^{2}+\left(\zeta-\zeta^{\prime}\right)^{2}\right]^{-1 / 2} \\
& \quad \times \delta\left(x^{\prime}-v_{x} t\right) \delta\left(y^{\prime}-v_{y} t\right) \delta\left(\sqrt{1-(v-w)^{2} / c_{o}^{2}} \zeta^{\prime}-v_{z} t\right) d x^{\prime} d y ' d \zeta^{\prime}
\end{aligned}
$$

Integrations in Eq. (21) and Eq. (17) yield for the EM potentials of the moving charge in $\Sigma(\mathbf{r}, \mathbf{t}, \mathbf{w})$ the solutions:

$$
\begin{gathered}
\mathbf{A}(\mathrm{x}, \mathrm{y}, \mathrm{z}, \mathrm{t})=\left[\mu_{\mathrm{o}} \mathrm{e}(\mathrm{v}-\mathrm{w}) / 4 \pi\right] /\left[\left(1-(\mathrm{v}-\mathrm{w})^{2} / \mathrm{c}_{\mathrm{o}}^{2}\right)\left\{\left(\mathrm{x}-\mathrm{v}_{\mathrm{x}} \mathrm{t}\right)^{2}+\left(\mathrm{y}-\mathrm{v}_{\mathrm{y}} \mathrm{t}\right)^{2}\right\}+\left(\mathrm{z}-\mathrm{v}_{\mathrm{z}} \mathrm{t}\right)^{2}\right]^{1 / 2} \\
\Phi(\mathrm{x}, \mathrm{y}, \mathrm{z}, \mathrm{t})=\left(\mathrm{e} / 4 \pi \epsilon_{\mathrm{o}}\right)\left[1+\mathrm{w} \bullet(\mathrm{v}-\mathrm{w}) / \mathrm{c}_{\mathrm{o}}^{2}\right] /\left[\left(1-(\mathrm{v}-\mathrm{w})^{2} / \mathrm{c}_{\mathrm{o}}^{2}\right)\left\{\left(\mathrm{x}-\mathrm{v}_{\mathrm{x}} \mathrm{t}\right)^{2}+\left(\mathrm{y}-\mathrm{v}_{\mathrm{y}} \mathrm{t}\right)^{2}\right\}+\left(\mathrm{z}-\mathrm{v}_{\mathrm{z}} \mathrm{t}\right)^{2}\right]^{1 / 2}
\end{gathered}
$$

In accordance with the Galilei covariant relations (4), Eqs. (22) and (23) give for the EM field of the charge (e) moving (v) uniformly in the ether flow w:

$$
\begin{aligned}
& \mathbf{E}(x, y, z, t)=\left(e / 4 \pi \epsilon_{o}\right)\left[1-(v-w)^{2} / c_{o}^{2}\right]\left\{\left[1+w \bullet(v-w) / c_{o}^{2}\right]\left[\left(x-v_{x} t\right) \mathbf{a}_{x}+\left(y-v_{y} t\right) \mathbf{a}_{y}\right]\right. \\
& \left.+\left[\left(z-v_{z} t\right)-\left(v_{z}-w_{z}\right)\left(v_{x}\left(x-v_{x} t\right)+v_{y}\left(y-v_{y} t\right)\right) / c_{o}^{2}\right] a_{z}\right\} / \\
& {\left[\left(1-(v-w)^{2} / c_{o}^{2}\right)\left\{\left(x-v_{x} t\right)^{2}+\left(y-v_{y} t\right)^{2}\right\}+\left(z-v_{z} t\right)^{2}\right]^{3 / 2},} \\
& \mathbf{H}(\mathrm{x}, \mathrm{y}, \mathrm{z}, \mathrm{t})=\left[\mathrm{e}\left(\mathrm{v}_{\mathrm{z}}-\mathrm{w}_{\mathrm{z}}\right) / 4 \pi\right]\left[1-(\mathrm{v}-\mathrm{w})^{2} / \mathrm{c}_{\mathrm{o}}^{2}\right]\left\{-\left(\mathrm{y}-\mathrm{v}_{\mathrm{y}} \mathrm{t}\right) \mathbf{a}_{\mathrm{x}}+\left(\mathrm{x}-\mathrm{v}_{\mathrm{x}} \mathrm{t}\right) \mathbf{a}_{\mathrm{y}}\right\} / \\
& {\left[\left(1-(v-w)^{2} / c_{o}^{2}\right)\left\{\left(x-v_{x} t\right)^{2}+\left(y-v_{y} t\right)^{2}\right\}+\left(z-v_{z} t\right)^{2}\right]^{3 / 2} .}
\end{aligned}
$$

Comparison shows that the EM fields of the uniformly moving (v) charge are interrelated for arbitrary ether velocities $w$ by

$$
\mathbf{H}=\epsilon_{\mathrm{o}}(\mathbf{v}-\mathbf{w}) \times \mathbf{E} /\left(1+\mathbf{w} \bullet(\mathbf{v}-\mathbf{w}) / \mathrm{c}_{\mathrm{o}}^{2}\right] .
$$

The solutions (22) - (25) are real throughout the entire space $|\mathbf{r}| \leq \infty$ if $|\mathbf{v}-\mathbf{w}|<c_{\mathrm{o}}$, whereas they are real only behind and imaginary ahead, of an advancing wave front if $|v-w|>c_{0}$. The latter discontinuous solutions are typical for hyperbolic equations. [24]

If $|\mathbf{v}-\mathbf{w}|<c_{o}$, the EM fields are continuous and the equipotential surfaces [see Eq. (23)] are the oblate ellipsoids of revolution centered at $\mathbf{r}=\mathbf{v t}$,

$$
\left(x-v_{x} t\right)^{2}+\left(y-v_{y} t\right)^{2}+\left(z-v_{z} t\right)^{2} /\left[1-(v-w)^{2} / c_{o}^{2}\right]=\text { const },>0 .
$$

The ellipsoid axes in the z-direction (parallel to v-w) are contracted by the factor $\left[1-(v-w)^{2} / c_{o}^{2}\right]^{1 / 2}$. If $|\mathbf{v}-\mathbf{w}|>c_{o}$, the EM fields are discontinuous and the equipotential surfaces [see Eq. (23)] are the hyperboloids of revolution,

$$
\left(x-v_{x} t\right)^{2}+\left(y-v_{y} t\right)^{2}-\left(z-v_{z} t\right)^{2} / \gamma^{2}=\text { const }>0
$$

about the $z^{\prime}$-axis (parallel to $v$-w) defined by $x=v_{x} t, y=v_{y} t$. The wave front is the cone of revolution about the $z^{\prime}$-axis with the vertex at $r=v t$,

$$
\left.\mathrm{v}_{\mathrm{z}} \mathrm{t}-\mathrm{z}=\gamma\left[\mathrm{x}-\mathrm{v}_{\mathrm{x}} \mathrm{t}\right)^{2}+\left(\mathrm{y}-\mathrm{v}_{\mathrm{y}} \mathrm{t}\right)^{2}\right]^{1 / 2}
$$

where

$$
\gamma=\left[(\mathbf{v}-\mathbf{w})^{2} / \mathrm{c}_{\mathrm{o}}^{2}-1\right]^{1 / 2}>0 .
$$

The fact that real EM field solutions exist for both $|\mathbf{v}-\mathbf{w}|<\mathrm{c}_{\mathrm{o}}$ and $|\mathrm{v}-\mathbf{w}|>\mathrm{c}_{\mathrm{o}}$ indicates that no electrodynamic limitation exists for the relative velocity $v-w$ between the charge $(v)$ and the ether (w). Relativistic restrictions are not applicable, since $\mathbf{v - w}$ is a Galilean relative velocity. [2] 
The fundamental equation (20) reveals that a moving charge excites a magnetic field $\mathbf{H}(\mathbf{r}, \mathbf{t})$ in $\Sigma(r, t, w)$ only if it moves relative to the ether, $\mathbf{v} \neq \mathbf{w}$. Therefore, charges in accelerated motion always excite magnetic fields in the uniformly streaming or resting ether (condition for radiation). The general electrodynamic solutions (22) - (26) imply other interesting physical conclusions, which shall be analyzed in simple examples.

Charge Moving with Ether Velocity. Assume that a charge e moves with a velocity $\mathbf{v}=\mathbf{w}$ $=\mathbf{v a}_{\mathbf{z}}$ in $\Sigma(\mathbf{r}, \mathbf{t}, \mathbf{w})$, so that the charge is at rest relative to the streaming ether, $\mathbf{v}-\mathbf{w}=\mathbf{0}$. In this case, the EM field of the moving charge is by Eqs. (24) and (25)

$$
\begin{gathered}
\mathbf{E}(\mathrm{r}, \mathrm{z}, \mathrm{t})=\left(\mathrm{e} / 4 \pi \epsilon_{\mathrm{o}}\right)\left\{\mathbf{r a}_{\mathrm{r}}+(\mathrm{z}-\mathrm{vt}) \mathbf{a}_{\mathrm{z}}\right\} /\left[\mathrm{r}^{2}+(\mathrm{z}-\mathrm{vt})^{2}\right]^{3 / 2}, \\
\mathbf{H}(\mathrm{r}, \mathrm{z}, \mathrm{t})=\mathbf{0},
\end{gathered}
$$

where $r=\left(x^{2}+y^{2}\right)^{1 / 2}$ since $w=w a_{z}$ (cylinder-symmetry about $z$-axis).

Equation (31) shows that the equipotential surfaces are given by $\mathrm{r}^{2}+(\mathrm{z}-\mathrm{vt})^{2}=$ const $>0$, i.e. these are spherical surfaces $r_{3}=$ const centered about the instantaneous position $r=0, z=v t$ of the charge. Thus, the Coulomb field configuration is not contracted, since the charge moving with the velocity $\mathbf{v}=\mathbf{w}$ experiences no ether flow.

Equation (32) indicates that a charge moving with a velocity $\mathbf{v}$ in an inertial frame $\Sigma(\mathbf{r}, \mathbf{t}, \mathbf{w})$ has no magnetic field, if the charge does not move relative to the ether $\left(\Sigma^{\circ}\right), \mathbf{v}=\mathbf{w}$. Hence, magnetic fields are excitations of the ether produced by charge motion relative to the ether.

At present, no comparison with experiments can be made, since the magnetic field of individual charges at rest in the ether has not been measured in inertial frames $\Sigma(r, t, w)$ when the charge velocity is large, $|\mathbf{v}| \lesssim \mathbf{c}_{\mathrm{o}}$. For small charge velocities, $|\mathbf{v}|<<\mathrm{c}_{\mathrm{o}}$, the corresponding STR field $\mathbf{H}=\epsilon_{\mathrm{o}} \mathbf{v} \times \mathbf{E} \propto \mathbf{O}$ is extremely small and approximates the Galilean field $\mathbf{H}=\mathbf{O}$ for $\mathbf{v}=\mathbf{w}$.

Charge at Rest in Ether Flow. Consider a charge e at rest $(v=0)$ at the origin $r=0$ of an inertial frame $\Sigma(\mathbf{r}, \mathbf{t}, \mathbf{w})$ with ether flow $\mathbf{w}=\mathbf{w a}_{\mathbf{z}}$. This charge moves with a velocity $-\mathbf{w}$ relative to the ether. By Eqs. (24) and (25), the EM field of this resting charge is in $\Sigma(r, t, w)$

$$
\begin{gathered}
\mathbf{E}(\mathrm{r}, \mathrm{z})=\left(\mathrm{e} / 4 \pi \epsilon_{\mathrm{o}}\right)\left(1-\mathrm{w}^{2} / \mathrm{c}_{\mathrm{o}}^{2}\right)\left\{\left(1-\mathrm{w}^{2} / \mathrm{c}_{\mathrm{o}}^{2}\right) \mathrm{ra}_{\mathrm{r}}+\mathrm{z \mathbf {a } _ { \mathrm { z } }}\right\} /\left[\left(1-\mathrm{w}^{2} / \mathrm{c}_{\mathrm{\sigma}}^{2}\right) \mathrm{r}^{2}+\mathrm{z}^{2}\right]^{3 / 2}, \\
\mathbf{H}(\mathrm{r}, \mathrm{z})=-(\mathrm{ew} / 4 \pi)\left(1-\mathrm{w}^{2} / \mathrm{c}_{\mathrm{o}}^{2}\right) r \mathbf{r}_{\boldsymbol{}} /\left[\left(1-\mathrm{w}^{2} / \mathrm{c}_{\mathrm{o}}^{2}\right) \mathrm{r}^{2}+\mathrm{z}^{2}\right]^{3 / 2},
\end{gathered}
$$

where $r, \phi, z$ are cylinder coordinates. Although the charge is at rest in $\Sigma(r, t, w)$ it has there a magnetic field $\mathbf{H}(\mathrm{r}, \mathrm{z})=-\epsilon_{\mathrm{o}} \mathbf{w} \times \mathbf{E}(\mathrm{r}, \mathrm{z}) /\left(1-\mathrm{w}^{2} / \mathrm{c}_{\mathrm{o}}^{2}\right)$, since the charge moves with a velocity $-\mathbf{w}$ relative to the ether (Galilei invariance of magnetic field, $\mathbf{H}=\mathbf{H}^{\circ}$ ).

By Eq. (33), the electric field is not radial in the ether flow $w$, since $E_{z} / E_{r}=z / r\left(1-w^{2} / c_{o}^{2}\right)$ * $z / r$. In particular, $E_{z} / E_{r} \rightarrow \infty$ for $w \rightarrow c_{o}$. Thus, the ether flow $w$ deforms the electric field configuration by convection.

The electric field component parallel to the ether flow $w$ along the ray $r=0$, in front $(z>0)$ and behind $(z<0)$ the charge at $r=0$,

$$
\mathrm{E}_{\mathrm{z}}(0, \mathrm{z})=\left(\mathrm{e} / 4 \pi \epsilon_{\mathrm{o}}\right)\left(1-\mathrm{w}^{2} / \mathrm{c}_{\mathrm{o}}^{2}\right) \mathrm{z} /|\mathrm{z}|^{3},
$$

is reduced by the ether flow factor $\left(1-w^{2} / c_{o}^{2}\right)$, with $E_{z}(0, z) \rightarrow 0$ for $w \rightarrow c_{o}$. The field component 
perpendicular to the ether flow $w$ and in the plane $z=0$ of the charge,

$$
E_{r}(r, 0)=\left(e / 4 \pi \epsilon_{o}\right)\left(1-w^{2} / c_{o}^{2}\right)^{1 / 2} / r^{2},
$$

decreases with increasing ether speed $w$, too, with $E_{r}(r, 0) \rightarrow 0$ for $w \rightarrow c_{o}$. On the other hand, Eq. (34) shows that the magnetic field in the charge plane $z=0$,

$$
H_{\phi}(r, 0)=-(e w / 4 \pi) r^{-2} /\left(1-w^{2} / c_{o}^{2}\right)^{1 / 2}
$$

increases with the ether speed $w$, with $-H_{\phi}(r, 0) \rightarrow \infty$ for $w \rightarrow c_{0}$. But, $H_{\phi}(r, z) \rightarrow 0$ in front $(z>0)$ and behind $(z<0)$ the charge for $w \rightarrow c_{o}$ by Eq. (34).

Accordingly, a magnetic field is excited not only by a charge moving relative to the ether, but also by an ether flow w relative to a charge at rest. Eq. (26) explains the magnetic field of a charge at rest as a magnetic induction by the ether flow across the electric flux density $\mathbf{D}=\epsilon_{\mathbf{o}} \mathbf{E}$.

On the earth, the ether velocity has been measured to be $w \sim 3 \times 10^{5} \mathrm{~m} / \mathrm{s}$, with $\mathrm{w} / \mathrm{c}_{\mathrm{o}} \sim 10^{-3}$, and $1-w^{2} / c_{o}^{2} \sim 1-10^{-6} \approx 1$. [21] For this reason, the interesting effects of electric field reduction and magnetic field excitation by the ether flow $w$ across a resting charge are small and difficult to ọbserve under terrestrial conditions.

Charge Moving in Ether Frame. An other limiting case is a charge moving with a velocity $\mathbf{v}=\mathbf{v} \mathbf{a}_{\mathbf{z}}$ in the ether frame $\Sigma^{0}=\Sigma(\mathbf{r}, \mathrm{t}, \mathbf{0})$, where $\mathbf{w} \equiv \mathbf{0}$. According to Eqs. (24) and (25), the EM fields of the moving charge are in $\Sigma(\mathbf{r}, \mathbf{t}, \mathbf{0})$ :

$$
\begin{gathered}
\left.\mathbf{E}(\mathrm{r}, \mathrm{z}, \mathrm{t})=\left(\mathrm{e} / 4 \pi \epsilon_{\mathrm{o}}\right)\left(1-\mathrm{v}^{2} / \mathrm{c}_{\mathrm{o}}^{2}\right)\left\{r \mathbf{a}_{\mathrm{r}}+(\mathrm{z}-\mathrm{vt}) \mathbf{a}_{\mathrm{z}}\right\} /\left(1-\mathrm{v}^{2} / \mathrm{c}_{\mathrm{o}}^{2}\right) \mathrm{r}^{2}+(\mathrm{z}-\mathrm{vt})^{2}\right]^{3 / 2} . \\
\mathbf{H}(\mathrm{r}, \mathrm{z}, \mathrm{t})=(\mathrm{ev} / 4 \pi)\left(1-\mathrm{v}^{2} / \mathrm{c}_{\mathrm{o}}^{2}\right) \mathbf{r a}_{\phi} /\left[\left(1-\mathrm{v}^{2} / \mathrm{c}_{\mathrm{o}}^{2}\right) \mathrm{r}^{2}+(\mathrm{z}-\mathrm{vt})^{2}\right]^{3 / 2} .
\end{gathered}
$$

These equations agree with the familiar EM field solutions for a uniformly moving charge of the ordinary Maxwell equations, which hold rigorously in the ether rest frame $\Sigma(r, t, 0)$. In particular, the fields are interrelated by $\mathbf{H}=\epsilon_{\mathrm{o}} \mathbf{v} \times \mathbf{E}$ (in accordance with Eq. (26) for $\mathbf{w}=\mathbf{0}$ ). Eq. (38) exhibits the Lorentz contraction of the equipotential surfaces in the directions parallel to $\mathbf{v}$, due to the absolute motion $\mathbf{v}$ of the charge relative to the ether.

Parallel Charge and Ether Velocities. The general solutions (22)-(25) for arbitrary velocity fields $\mathbf{v}=\left\{\mathbf{v}_{\mathrm{x}}, \mathbf{v}_{\mathbf{y}}, \mathrm{v}_{\mathbf{z}}\right\}$ and $\mathbf{w}=\left(\mathbf{w}_{\mathbf{x}}, \mathbf{w}_{\mathbf{y}}, \mathbf{w}_{\mathbf{z}}\right\}$ assume a particularly simple form if $\mathbf{v}$ and $\mathbf{w}$ are parallel, $\mathbf{v}=\mathbf{v} \mathbf{a}_{\mathbf{z}}$ and $\mathbf{w}=\mathbf{w} \mathbf{a}_{\mathbf{z}}$. The EM fields of the moving charge (v) in the ether flow (w) are for $\mathbf{v} \|$ w:

$$
\begin{gathered}
\Phi(r, z, t)=\left(e / 4 \pi \epsilon_{\mathrm{o}}\right)\left[1+\mathrm{w} \bullet(\mathrm{v}-\mathrm{w}) / \mathrm{c}_{\mathrm{o}}^{2} \mathrm{~g} /\left[\left(1-(\mathrm{v}-\mathrm{w})^{2} / \mathrm{c}_{\mathrm{o}}^{2}\right) \mathrm{r}^{2}+(\mathrm{z}-\mathrm{vt})^{2}\right]^{1 / 2},\right. \\
\mathbf{A}(\mathrm{r}, \mathrm{z}, \mathrm{t})=\left[\mu_{\mathrm{o}} \mathrm{e}(\mathrm{v}-\mathrm{w}) / 4 \pi\right] /\left[\left(1-(\mathrm{v}-\mathrm{w})^{2} / \mathrm{c}_{\mathrm{o}}^{2}\right) \mathrm{r}^{2}+(\mathrm{z}-\mathrm{vt})^{2}\right]^{1 / 2},
\end{gathered}
$$

and

$$
\begin{gathered}
\mathbf{E}(\mathrm{r}, \mathrm{z}, \mathrm{t})=\left(\mathrm{e} / 4 \pi \epsilon_{\mathrm{o}}\right)\left[1-(\mathrm{v}-\mathrm{w})^{2} / \mathrm{c}_{\mathrm{o}}^{2}\right]\left\{\left[1+\mathrm{w} \bullet(\mathrm{v}-\mathrm{w}) / \mathrm{c}_{\mathrm{o}}^{2}\right] \mathbf{a}_{\mathrm{r}}+(\mathrm{z}-\mathrm{vt}) \mathbf{a}_{\mathrm{z}}\right\} /\left[\left(1-(\mathrm{v}-\mathrm{w})^{2} / \mathrm{c}_{\mathrm{o}}^{2}\right) \mathrm{r}^{2}+(\mathrm{z}-\mathrm{vt})^{2}\right]^{3 / 2},(42) \\
\mathbf{H}(\mathrm{r}, \mathrm{z}, \mathrm{t})=[\mathrm{e}(\mathrm{v}-\mathrm{w}) / 4 \pi]\left[1-(\mathrm{v}-\mathrm{w})^{2} / \mathrm{c}_{\mathrm{o}}^{2}\right] \mathbf{a}_{\phi} /\left[\left(1-(\mathrm{v}-\mathrm{w})^{2} / \mathrm{c}_{\mathrm{o}}^{2}\right) \mathrm{r}^{2}+(\mathrm{z}-\mathrm{vt})^{2}\right]^{3 / 2}
\end{gathered}
$$

The cases discussed above are readily understood as special solutions of Eqs. (40)-(43).

In conclusion, it is noted the electrodynamics of a moving charge in vacuum under arbitrary conditions $(\mathbf{v} \neq \mathbf{w}$ or $\mathbf{v}=\mathbf{w})$ does not impose a restriction on the relative velocity $\mathbf{v}-\mathbf{w}$ between a 
charged particle (v) and the EM ether (w). Since $\mathbf{v}$ and $\mathbf{w}$ are defined in the same inertial frame $\Sigma(\mathbf{r}, \mathbf{t}, \mathbf{w})$, the Galilean relative velocity $\mathbf{v}-\mathbf{w}$ is not bounded by the limitation $\left|\mathbf{v}_{1}-\mathbf{v}_{2}\right|<c_{o}$ for the relativistic relative velocity $\mathbf{v}_{1}-\mathbf{v}_{2}$ (nonlinear addition theorem of STR) [2] of two systems.

\section{EM SHOCK WAVES IN SUBSTRATUM}

The generation of EM shock waves in an inertial frame $\Sigma(r, t, w)$ with ether flow $w$ requires superluminal electron velocities $\mathbf{v}-\mathbf{w}$ relative to the ether, $|\mathbf{v}-\mathbf{w}|>c_{0}$ by Eq. (30). Since $\mathbf{v}-\mathbf{w}$ is a Galilean relative velocity, the condition $|v-w|>c_{0}$ is realizable by first principles. Even if both $|\mathbf{v}|<\mathrm{c}_{\mathrm{o}}$ and $|\mathbf{w}|<\mathrm{c}_{\mathrm{o}}$ are subluminal, the requirement $|\mathbf{v}-\mathbf{w}|>\mathrm{c}_{\mathrm{o}}$ can be satisfied if $\mathbf{v}$ and $\mathbf{w}$ are antiparallel, such that $|\mathbf{v}|>c_{o}-|w|$. However, since accelerator experiments have not yet been interpreted by means of Galilean relativity theory, we propose to discuss the possibility of EM shock waves in vacuum based on the hypothesis that superluminal electron velocities $\left|v_{1}-v_{2}\right|>c_{o}$ relative to the ether might be achievable. The existence of superluminal relative Galilean velocities $\left|\mathbf{v}_{1}-\mathbf{v}_{2}\right|$ $>\mathrm{c}_{\mathrm{o}}$ between ordinary systems is an experimental fact, e.g., the relative velocity between two subluminal electron beams with velocities $v_{1,2}= \pm 0.9 c_{o}$ in opposite directions is measured to be $v_{1}-v_{2}=1.8 c_{o}>c_{o}$ in the frame of reference in which these Galilean velocities are represented. [2]

For superluminal relative Galilean velocities v-w between the moving charge (v) and the ether (w), the critical parameter $\gamma=\left[(v-w)^{2} / c_{o}^{2}-1\right]^{1 / 2}$ is (positive) real, Eq. (30). Based on the hypothesis of superluminal relative velocities $v-w$, the EM field solutions (22)-(25) are rewritten first in the form of shock solutions with a discontinuous wave front, ahead of which the ether is not perturbed:

$$
\begin{gathered}
A(r, t)=\left[\mu_{0} e(v-w) / 4 \pi\right] /\left[\left(z-v_{z} t\right)^{2}-\gamma^{2}\left(x-v_{x} t\right)^{2}-\gamma^{2}\left(y-v_{y} t\right)^{2}\right]^{1 / 2}, \quad v_{z} t-z>\gamma\left[\left(x-v_{x} t\right)^{2}+\left(y-v_{y} t\right)^{2}\right]^{1 / 2}, \\
=0, \quad v_{z} t-z<\gamma\left[\left(x-v_{x} t\right)^{2}+\left(y-v_{y} t\right)^{2}\right]^{1 / 2} ; \\
\Phi(r, t)=\left(e / 4 \pi \epsilon_{0}\right)\left[1+w \bullet(v-w) / c_{0}^{2}\right] /\left[\left(z-v_{z} t\right)^{2}-\gamma^{2}\left(x-v_{x} t\right)^{2}-\gamma^{2}\left(y-v_{y} t\right)^{2}\right]^{1 / 2}, \\
\quad v_{z} t-z>\gamma\left[\left(x-v_{x} t\right)^{2}+\left(y-v_{y} t\right)^{2}\right]^{1 / 2}, \\
=0, \quad v_{z} t-z<\gamma\left[\left(x-v_{x} t\right)^{2}+\left(y-v_{y} t\right)^{2}\right]^{1 / 2} ;
\end{gathered}
$$

and

$$
\begin{gathered}
\mathbf{E}(\mathbf{r}, \mathrm{t})=-\left(e / 4 \pi \epsilon_{\mathrm{o}}\right) \gamma^{2}\left\{\left[1+w \bullet(v-w) / c_{o}^{2}\right]\left[\left(x-v_{x} t\right) \mathbf{a}_{x}+\left(y-v_{y} t\right) \mathbf{a}_{y}\right]+\left[\left(z-v_{z} t\right)-\left(v_{z}-w_{z}\right)\left(v_{x}\left(x-v_{x} t\right)\right.\right.\right. \\
\left.\left.\left.+v_{y}\left(y-v_{y} t\right)\right) / c_{o}^{2}\right] \mathbf{a}_{z}\right\} / N(r, t)^{3}, \quad v_{z} t-z>\gamma\left[\left(x-v_{x} t\right)^{2}+\left(y-v_{y} t\right)^{2}\right]^{1 / 2}, \\
=0, \quad v_{z} t-z<\gamma\left[\left(x-v_{x} t\right)^{2}+\left(y-v_{y} t\right)^{2}\right]^{1 / 2} ; \\
\mathbf{H}(\mathbf{r}, t)=-\left[e\left(v_{z}-w_{z}\right) / 4 \pi\right] \gamma^{2}\left\{-\left(y-v_{y} t\right) \mathbf{a}_{x}+\left(x-v_{x} t\right) \mathbf{a}_{y}\right\} / N(r, t)^{3}, \quad v_{z} t-z>\gamma\left[\left(x-v_{x} t\right)^{2}+\left(y-v_{y} t\right)^{2}\right]^{1 / 2}, \\
=0, \quad v_{z} t-z<\gamma\left[\left(x-v_{x} t\right)^{2}+\left(y-v_{y} t\right)^{2}\right]^{1 / 2} ;
\end{gathered}
$$

where

$$
N(r, t)=\left[\left(z-v_{z} t\right)^{2}-\gamma^{2}\left(x-v_{x} t\right)^{2}-\gamma^{2}\left(y-v_{y} t\right)^{2}\right]^{1 / 2}
$$

and $\gamma$ is defined in Eq. (30). For any given time $t>0$, the equipotential surfaces $\Phi(r, t)=$ const are the hyperboloids of revolution (28). The wave front at time $t>0$ is the cone of revolution (29) 
about the line $\left(x=v_{x} t, y=v_{y} t\right)$ with the vertex at the charge position $r=v t$. The singular discontinuity at the shock front, $v_{z} t-z=\gamma\left[\left(x-v_{x} t\right)^{2}+\left(y-v_{y} t\right)^{2}\right]^{1 / 2}$, can be shown to be caused by the point charge model.

The EM shock solutions (44)-(47) are geometrically complicated for arbitrary velocity fields $\mathbf{v}=\left\{\mathbf{v}_{\mathbf{x}}, \mathbf{v}_{\mathbf{y}}, \mathbf{v}_{\mathbf{z}}\right\}$ and $\mathbf{w}=\left\{\mathbf{w}_{\mathbf{x}}, \mathbf{w}_{\mathbf{y}}, \mathbf{w}_{\mathbf{z}}\right\}$. For this reason, let the most important case of parallel charge and ether velocities, $\mathbf{v}=\mathbf{v a}_{\mathbf{z}}, \mathbf{w}=\mathbf{w a}_{\mathbf{z}}$, be discussed in more detail. In cylindrical coordinates $(\mathrm{r}, \phi, \mathrm{z})$, Eqs. (44)-(47) become for $\mathrm{v}|| \mathrm{w}$ :

$$
\begin{aligned}
& A(r, z, t)=\left[\mu_{0} e(v-w) / 4 \pi\right] /\left[(v t-z)^{2}-\gamma^{2} r^{2}\right]^{1 / 2}, \quad v t-z>\gamma r, \\
& =0, \quad v t-z<\gamma r ; \\
& \Phi(r, z, t)=\left(e / 4 \pi \epsilon_{o}\right)\left[1+w \bullet(v-w) / c_{o}^{2}\right] /\left[(v t-z)^{2}-\gamma^{2} r^{2}\right]^{1 / 2}, \quad v t-z>\gamma r, \\
& =0, \quad \mathrm{vt}-\mathrm{z}<\gamma \mathrm{r}
\end{aligned}
$$

and

$$
\begin{gathered}
\mathbf{E}(\mathrm{r}, \mathrm{z}, \mathrm{t})=-\left(\mathrm{e} / 4 \pi \epsilon_{\mathrm{o}}\right) \gamma^{2}\left\{\left[1+\mathrm{w} \bullet(\mathrm{v}-\mathrm{w}) / \mathrm{c}_{\mathrm{o}}^{2}\right] \mathrm{ra}+(\mathrm{z}-\mathrm{vt}) \mathbf{a}_{\mathrm{z}}\right\} /\left[(\mathrm{vt}-\mathrm{z})^{2}-\gamma^{2} \mathrm{r}^{2}\right]^{3 / 2}, \mathrm{vt}-\mathrm{z}>\gamma \mathrm{r}, \\
=0, \quad \mathrm{vt}-\mathrm{z}<\gamma \mathrm{r} ; \\
\begin{aligned}
\mathbf{H}(\mathrm{r}, \mathrm{z}, \mathrm{t})=-[\mathrm{e}(\mathrm{v}-\mathrm{w}) / 4 \pi] \gamma^{2} \mathrm{ra} /\left[(\mathrm{vt}-\mathrm{z})^{2}-\gamma^{2} \mathrm{r}^{2}\right]^{3 / 2}, \quad \mathrm{vt}-\mathrm{z}>\gamma \mathrm{r}, \\
=0, \quad \mathrm{vt}-\mathrm{z}<\gamma \mathrm{r} .
\end{aligned}
\end{gathered}
$$

It is recognized that the EM shock fields exist only behind the shock front where the fields drop discontinuously to zero. The equipotential surfaces [see Eq. (50)] are the hyperboloids of revolution inside the wave front cone (FIG. 2)

$$
\mathrm{r}^{2}-(\mathrm{z}-\mathrm{vt})^{2} / \gamma^{2}=\text { const }>0,
$$

about the $\mathrm{z}$-axis (parallel to $\mathrm{v}$-w). Eq. (53) gives $\mathrm{r}= \pm(\mathrm{z}$-vt) $/ \gamma$ for const $=0$, i.e., the $\mathrm{EM}$ wave front is the cone of revolution about the z-axis,

$$
v t-z=\gamma r
$$

with

$$
\operatorname{tg} \theta=r /(v t-z)=\gamma^{-1}
$$

the relation for the half-angle $\theta$ of the cone (FIG. 2). The conical wave front (54) is the envelope of spherical EM waves originating from the "past" positions $(r=0, z=v t)$ of the charge. At time $t>0$, the charge $e$ is at $z=v t$ (vertex of cone, FIG. 2).

The optimum experimental arrangement for the observation of EM shock waves in vacuum would be an electron beam with a velocity $\mathbf{v}$, which is antiparallel to the terrestrial ether velocity $w \sim 3 \times 10^{5} \mathrm{~m} / \mathrm{s}$. In this case, Eqs. (50) and (51) yields for the electron beam the EM shock condition $|\mathrm{v}|>\mathrm{c}_{\mathrm{o}}-|\mathrm{w}| \equiv \mathrm{v}_{\mathrm{cr}}$. With $\mathrm{w}=3 \times 10^{5} \mathrm{~m} / \mathrm{s}$ at the earth, the required beam speed would be $\mathrm{v}>$ $2.997925 \times 10^{8}\left(1-10^{-3}\right) \mathrm{m} / \mathrm{s}$.

The general condition for the excitation of the EM shock waves in the case of arbitrary charge and either velocities is $|v-w|>c_{o}$ by Eqs. (44)-(47), i.e., the charge has to move with a Galilean velocity $\mathbf{v - w}$ relative to the ether $(w)$ which exceeds the characteristic wave speed $c_{o}$ of the 
ether. Accordingly, the discontinuous EM waves represent shock waves in the ether. Experimental verification of the EM shock waves in vacuum would provide direct evidence for the existence of the EM wave carrier (ether).

\section{VACUUM CERENKOV EFFECT}

Based on the hypothesis of superluminal Galilean charge velocities $|v-w|>c_{0}$ relative to the ether, a spectral theory of the Cerenkov radiation emitted by a moving charge in vacuum is given. As a result of the radiative energy loss, the velocity of the charge would decrease, $|v-w| \rightarrow$ $c_{0}$. To simplify the analysis, the charge velocity is assumed to be quasi-constant, requiring that the charge does not slow down too rapidly, $\mathrm{T}|\mathrm{dv} / \mathrm{dt}|<\mathrm{c}_{\mathrm{o}}$, in a period $\mathrm{T}=2 \pi / \omega$ of the respective wave of frequency $\omega$.

EM Radiation Fields. For the observation of the vacuum Cerenkov effect, antiparallel charge and ether velocities are optimum, $v=v a_{z}$ and $w=w a_{2}$ where sign $w=-s i g n v$. For this reason, and mathematical simplification, we assume $\mathbf{v}$ parallel $\mathbf{w}$. The current density of the charge relative to the ether is the source of the EM radiation. By Eqs. (1) and (2), the vectory potential $A=A(r, z, t) a_{2}$ and scalar potential $\Phi=\Phi(r, z, t)$ of the EM radiation are determined by $[r, \phi, z$ cylinder coordinates with $\left.\mathbf{a}_{\mathbf{z}} \|(\mathbf{v}-\mathbf{w})\right]$

$$
\begin{gathered}
{\left[\mu_{\mathrm{o}} \epsilon_{\mathrm{o}}(\partial / \partial t+w \bullet \nabla)^{2}-\nabla^{2}\right] \mathrm{A}=\mu_{\mathrm{o}} \mathrm{e}[\delta(\mathrm{r}) / 2 \pi \mathrm{r}] \delta(\mathrm{z}-\mathrm{vt})(\mathrm{v}-\mathrm{w}),} \\
\Phi=\left[1+\mathrm{w} \bullet(\mathrm{v}-\mathrm{w}) / \mathrm{c}_{\mathrm{o}}^{2}\right] \mathrm{A} / \mu_{\mathrm{o}} \epsilon_{\mathrm{o}}(\mathrm{v}-\mathrm{w}) .
\end{gathered}
$$

By means of the operator identity (18) for uniform charge motion, Eq. (56) is reduced to the Poisson equation with $\mathbf{r}, \mathbf{t}$-dependent source,

$$
\left[\mathrm{r}^{-1}(\partial / \partial \mathrm{r})(\mathrm{r} \partial / \partial \mathrm{r})+\left(1-(\mathrm{v}-\mathrm{w})^{2} / \mathrm{c}_{\mathrm{o}}^{2}\right) \partial^{2} / \partial z^{2}\right] \mathrm{A}=-\mu_{\mathrm{o}} \mathrm{e}[\delta(\mathrm{r}) / 2 \pi \mathrm{r}] \delta(\mathrm{z}-\mathrm{vt})(\mathrm{v}-\mathrm{w}) .
$$

In order to find the spectral amplitude $\mathbf{A}(\mathbf{r}, \boldsymbol{\omega})$ of the vector potential $\mathbf{A}(\mathbf{r}, \mathbf{t})$,

$$
A(r, \omega)=(1 / 2 \pi) \int_{-\infty}^{+\infty} A(r, t) e^{i \omega t} d t,
$$

Eq. (58) is Fourier transformed in accordance with Eq. (59), with the result

$$
\begin{gathered}
{\left[\mathrm{r}^{-1}(\partial / \partial \mathrm{r})(\mathrm{r} \partial / \partial \mathrm{r})+\partial^{2} / \partial \zeta^{2}\right] \mathrm{A}(\mathrm{r}, \zeta, \omega)=-\left(\mu_{\mathrm{o}} \mathrm{e} / 2 \pi\right)[\delta(\mathrm{r}) / 2 \pi \mathrm{r}](\mathrm{v}-\mathrm{w})} \\
\times \int_{-\infty}^{+\infty} \delta\left(\sqrt{1-(\mathrm{v}-\mathrm{w})^{2} / \mathrm{c}_{\mathrm{o}}^{2}} \zeta-\mathrm{vt}\right) \mathrm{e}^{\mathrm{i} \omega \mathrm{t}} \mathrm{dt}
\end{gathered}
$$

where $\zeta(\mathrm{z})$ is the "dilated" axial coordinate defined in Eq. (20). Evaluation of the integral and substitution of

$$
A(r, \zeta, \omega)=\left(\mu_{0} e / 2 \pi v\right)(v-w)\left\{\exp \left[i \omega \sqrt{1-(v-w)^{2} / c_{0}^{2}} \zeta / v\right]\right\} F(r, \omega)
$$

reduces Eq. (60) to a Helmholtz equation for the radial amplitude function $F(r, \omega)$,

$$
\left[r^{-1}(\partial / \partial r)(r \partial / \partial r)+\hat{k}^{2}\right] F(r, \omega)=-\delta(r) / 2 \pi r
$$

where

$$
\hat{\mathbf{k}}^{2}=\left(\omega^{2} / c_{\mathrm{o}}^{2}\right)\left[(\mathrm{v}-\mathrm{w})^{2} / \mathrm{v}^{2}\right]\left[1-\mathrm{c}_{\mathrm{o}}^{2} /(\mathrm{v}-\mathrm{w})^{2}\right]>0, \quad|\mathrm{v}-\mathrm{w}|>\mathrm{c}_{\mathrm{o}} .
$$


Since the source is singular at $r=0$, and the solutions of Eq. (62) have to represent outgoing waves, $F(r, \omega)$ is proportional to the Hankel function of the 1-st kind and 0-th order (singular at $r$ $=0)$,

$$
\left.\mathrm{F}(\mathrm{r}, \boldsymbol{\omega})=\mathrm{CH}_{\mathrm{o}}^{(\mathbf{l})} \hat{\mathrm{k}} \mathrm{r}\right) ; \quad \mathrm{F}(\mathrm{r}, \boldsymbol{\omega}) \rightarrow 0, \mathrm{r} \rightarrow \infty .
$$

Integration of Eq. (62) over the area $2 \pi$ rdr gives in the limit $r \rightarrow 0$ the constant $C$,

$$
\lim _{r \rightarrow 0} 2 \pi \mathrm{r} d\left[\mathrm{CH}_{\mathrm{o}}^{(1)}(\hat{\mathrm{k}} \mathrm{r})\right] / \mathrm{dr}=-1, \mathrm{C}=\mathrm{i} / 4,
$$

since $H_{o}^{(1)}(\hat{k} r) \sim i(2 / \pi) \ell n(\hat{k} r)$ for $r \rightarrow 0$. Accordingly,

$$
F(r, \omega)=(i / 4) H_{o}^{(1)}(\hat{k} r) \text {. }
$$

By Eqs. (61) and (57), the Fourier amplitudes of the EM potentials are:

$$
\begin{gathered}
\mathbf{A}(\mathrm{r}, \mathrm{z}, \boldsymbol{\omega})=\mathrm{i}\left(\mu_{\mathrm{o}} \mathrm{e} / 8 \pi \mathrm{v}\right)(\mathbf{v}-\mathbf{w}) \mathrm{H}_{\mathrm{o}}^{(1)}(\hat{\mathrm{k}} \mathrm{r}) \mathrm{e}^{\mathrm{i} \omega z / N}, \\
\Phi(r, z, \omega)=\mathrm{i}\left(\mathrm{e} / 8 \pi \epsilon_{\mathrm{o}} \mathrm{v}\right)\left[1+w \bullet(\mathbf{v}-\mathbf{w}) / \mathrm{c}_{0}^{2}\right] \mathrm{H}_{\mathrm{o}}^{(1)}(\hat{\mathrm{k}} \mathrm{r}) \mathrm{e}^{\mathrm{i} \omega z / N} .
\end{gathered}
$$

The Fourier amplitudes of the EM fields $\mathbf{H}(\mathbf{r}, \boldsymbol{\omega})=\nabla \times \mathbf{A}(\mathbf{r}, \boldsymbol{\omega}) / \mu_{\mathrm{o}}$ and $\mathbf{E}(\mathbf{r}, \boldsymbol{\omega})=-\nabla \Phi(\mathbf{r}, \boldsymbol{\omega})+i \boldsymbol{i} \mathbf{A}(\mathbf{r}, \boldsymbol{\omega})$ follow from Eqs. (67) and (68),

$$
\begin{gathered}
H_{\phi}(r, z, \omega)=i(e / 8 \pi v)(v-w) \hat{k} H_{1}^{(1)}(\hat{k} r) e^{1 \omega z / N} \\
E_{r}(r, z, \omega)=i\left(e / 8 \pi \epsilon_{o} v\right)\left[1+w \bullet(v-w) / c_{o}^{2}\right] \hat{k} H_{1}^{(1)}(\hat{k} r) e^{i \omega z / N}, \\
E_{z}(r, z, \omega)=-\left(e / 8 \pi \epsilon_{o} v\right)(\omega / v) \gamma^{2} H_{o}^{(1)}(\hat{k} r) e^{1 \omega z / N},
\end{gathered}
$$

where $\hat{\mathbf{k}}=\hat{\mathbf{k}}(\omega)$, Eq. (63), and $\boldsymbol{\gamma}>0$, Eq. (30).

For the evaluation of the power radiated by the moving charge, the EM fields in the radiation zone, $\hat{\mathrm{k}} \mathrm{r}>>1$, are required. With,

$$
\begin{aligned}
& \mathrm{H}_{\mathrm{o}}^{(1)}(\hat{\mathrm{k}} \mathrm{r}) \sim(2 / \mathrm{i} \pi \hat{\mathbf{k}} \mathbf{r})^{1 / 2} \mathrm{e}^{\hat{i} \mathbf{k} r}, \hat{\mathbf{k}} \mathrm{r}>>1, \\
& \mathrm{H}_{1}^{(1)}(\hat{\mathrm{k}} \mathrm{r}) \sim-\mathrm{i}(2 / \mathrm{i} \pi \hat{\mathrm{k}} \mathrm{r})^{1 / 2} \mathrm{e}^{\hat{\mathrm{i}} \mathrm{r}}, \hat{\mathbf{k}} \mathrm{r}>>1 \text {, }
\end{aligned}
$$

we find that the EM field represents an axially symmetric wave in the radiation zone, with wave vector $\mathbf{k}=\hat{\mathbf{k}} \mathbf{a}_{\mathbf{r}}+(\boldsymbol{\omega} / \mathbf{v}) \mathbf{a}_{\mathbf{z}}$ :

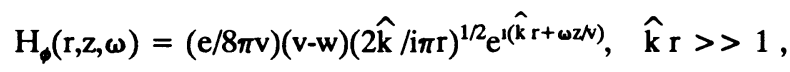

$$
\begin{aligned}
& \mathrm{E}_{\mathrm{r}}(\mathrm{r}, \mathrm{z}, \omega)=\left(\mathrm{e} / 8 \pi \epsilon_{\mathrm{o}} \mathrm{v}\right)\left[1+\mathrm{w} \bullet(\mathrm{v}-\mathrm{w}) / \mathrm{c}_{\mathrm{o}}^{2}\right](2 \hat{\mathrm{k}} / \mathrm{i} \pi \mathrm{r})^{1 / 2} \mathrm{e}^{\mathrm{i}(\hat{\mathrm{k}} \mathrm{r}+\omega z / v)}, \hat{\mathrm{k}} \mathrm{r}>>1, \\
& E_{z}(r, z, \omega)=-\left(e / 8 \pi \epsilon_{0} v\right)(\omega / \hat{k} v) \gamma^{2}(2 \hat{k} / i \pi r)^{1 / 2} e^{i(\hat{k} r+\omega z / v)}, \quad \hat{k} r>>1 .
\end{aligned}
$$

These radiated EM waves exist only if $\hat{k}$ is real, i.e. [Eq. (63)], if the Galilean velocity v-w of the charge relative to the ether is (radiation condition)

$$
|\mathbf{v}-\mathbf{w}|>c_{o} \text {. }
$$

As expected, the condition for Cerenkov radiation in vacuum agrees with that for EM shock waves in vacuum. For $|\mathbf{v}-\mathbf{w}|<c_{o}, \hat{k}(\omega)$ is imaginary, i.e. the waves are rapidly attenuated.

The planes of constant phase or amplitude state of the emitted waves, ker = const, are given by

$$
\hat{\mathbf{k}} \mathbf{r}+(\omega / \mathrm{v}) \mathrm{z}=\text { const }
$$


The radiation is emitted in the direction of the wave vector $\mathbf{k}=\{\hat{\mathrm{k}}, 0, \boldsymbol{\omega} / \mathrm{v}\}$, i.e., under the angle $\boldsymbol{\theta}_{\mathrm{o}}$ with the line of motion (z-axis) of the charge, given by (FIG. 2)

$$
\cos \theta_{\mathrm{o}}=\mathrm{c}_{\mathrm{o}}|\mathbf{v}-\mathbf{w}|<1,|\mathbf{v}-\mathbf{w}|>\mathrm{c}_{\mathrm{o}} .
$$

Since $\mathbf{E} \perp \mathbf{H}_{\boldsymbol{\phi}}$, the electric field lies in the plane spanned by the vectors $\mathbf{v}$ and $\mathbf{k}$ (polarization plane).

Radiated EM Energy. The EM energy flow per unit area and time is given by the Poynting vector $\mathbf{P}=\mathbf{E} \times \mathbf{H}\left[\mathrm{J} / \mathrm{m}^{2} \mathrm{~s}\right]$. The energy radiated through a cylinder surface $2 \pi R L$ at a distance $r$ (within the radiation zone, $\hat{\mathrm{k}} \mathrm{r}>>1$ ) from the charge trajectory (z-axis) is

$$
\mathrm{W}=\operatorname{Re} 2 \pi \mathrm{r} \int_{0}^{\mathrm{L}} \mathrm{dz} \int_{-\infty}^{+\infty}\left[-\mathrm{E}_{\mathrm{z}}(\mathrm{r}, \mathrm{t}) \mathrm{H}_{\odot}(\mathrm{r}, \mathrm{t})\right] \mathrm{dt} .
$$

where

$$
\begin{gathered}
E_{z}(r, t)=-\left(e / 8 \pi v^{2}\right)(2 / \pi r)^{1 / 2} \int_{-\infty}^{+\infty} \gamma(\omega)^{2} \epsilon_{o}^{-1}(\omega) \omega \hat{k}(\omega)^{-1 / 2} e^{-i(\omega t-\hat{k}(\omega) r-\omega z / v+\pi / 4)} d \omega, \hat{k} r>>1, \\
H_{\phi}(r, t)=(e / 8 \pi v)(v-w)(2 / \pi r)^{1 / 2} \int_{-\infty}^{+\infty} \hat{k}(\omega)^{1 / 2} e^{-i(\omega t-\hat{k}(\omega) r-\omega z / v+\pi / 4)} d \omega, \hat{k} r>>1,
\end{gathered}
$$

in complex representation by Eqs. (74) and (76), and the inverse Fourier integral theorem. Because of the physical cut-off at a maximum frequency $\omega=\omega_{0}, \epsilon_{0}(\omega)$ and $\mu_{\mathrm{o}}(\omega)$ in $\gamma(\omega)$ and $\hat{\mathrm{k}}(\omega)$ of Eqs. (81)-(82) are treated like discontinuous functions of $\omega$ in the mathematical frequency space $-\infty \leq$ $\omega \leq+\infty$.

Combining of Eqs. (80)-(82) yields for the radiative energy pulse in $|t| \leq \infty$,

$$
\begin{aligned}
\mathrm{W}= & \frac{1}{2}(\mathrm{e} / 4 \pi \mathrm{v})^{2}|(\mathrm{v}-\mathrm{w}) / \mathrm{v}| \int_{0}^{\mathrm{L}} \mathrm{dz} \int_{-\infty}^{+\infty} \mathrm{d} \omega \int_{-\infty}^{+\infty} \mathrm{d} \omega^{\prime} \gamma(\omega)^{2} \epsilon_{\mathrm{o}}^{-1}(\omega) \omega \hat{\mathrm{k}}(\omega)^{-1 / 2} \\
& \times \hat{\mathrm{k}}\left(\omega^{\prime}\right)^{1 / 2} \operatorname{exp~i}\left\{\left[\hat{\mathrm{k}}(\omega)-\hat{\mathrm{k}}\left(\omega^{\prime}\right)\right] \mathrm{r}+\left(\omega-\omega^{\prime}\right) \mathrm{z} / \mathrm{v}\right\} \int_{-\infty}^{+\infty} \mathrm{e}^{-\mathrm{i}\left(\omega-\omega^{\prime}\right) \mathrm{t}_{\mathrm{dt}}}
\end{aligned}
$$

where

$$
\int_{-\infty}^{+\infty} \mathrm{e}^{-\mathrm{i}\left(\boldsymbol{\omega}-\boldsymbol{\omega}^{\prime}\right) \mathrm{t}} \mathrm{dt}=2 \pi \delta\left(\boldsymbol{\omega}-\boldsymbol{\omega}^{\prime}\right)
$$

and $\hat{\mathrm{k}}(\omega)^{2} \propto \omega^{2}$ for $0 \leq \omega \leq \omega_{0}$. By Eq. (84), the r, z-dependence of the integrand is eliminated by the $\mathrm{d} \omega^{\prime}$-integration. Thus, we find for the radiated energy of the uniformly moving charge in vacuum:

$$
\mathrm{W}=\mathrm{L}\left(\mathrm{e}^{2} / 4 \pi \mathrm{v}^{2}\right)|(\mathrm{v}-\mathrm{w}) / \mathrm{v}| \int_{0}^{\infty} \epsilon_{\mathrm{o}}(\omega)^{-1}\left[(\mathrm{v}-\mathrm{w})^{2} / \mathrm{c}_{\mathrm{o}}(\omega)^{2}-1\right] \omega \mathrm{d} \omega
$$

or

$$
\mathrm{W}=\mathrm{L}\left(\mu_{\mathrm{o}} \mathrm{e}^{2} / 4 \pi\right)|(\mathrm{v}-\mathrm{w}) / \mathrm{v}|^{3} \int_{0}^{\omega} \mathrm{\omega}\left[1-c_{j}^{2} /(\mathrm{v}-\mathrm{w})^{2}\right] \omega \mathrm{d} \omega .
$$

If $|\mathrm{v}-\mathrm{w}|>\mathrm{c}_{\mathrm{o}}$, then $\mathrm{W}>0$, i.e., the charged particle excites radiation and is deaccelerated. Quantitatively, this radiation is small for one charge, but significant for an intense beam of charges.

For coherence reasons, the upper limit $\omega_{0}=2 \pi c_{\alpha} / \lambda_{o}$ of the radiation spectrum is determined by the radius $r_{o}=e^{2} / 4 \pi \epsilon_{0} m c_{o}^{2}$ of the charged particle $(e, m)$, which imposes a minimum wave length $\lambda_{\mathrm{o}}=2 \pi \mathrm{r}_{\mathrm{o}}$. E.g., for an electron $\mathrm{r}_{\mathrm{e}}=2.8 \times 10^{-15} \mathrm{~m}$ and $\omega_{\mathrm{o}}=\mathrm{c}_{\mathrm{o}} / \mathrm{r}_{\mathrm{o}} \sim 10^{23} \mathrm{~s}^{-1}$. Thus the cut-off for the electron Cerenkov effect in vacuum is in the $\gamma$-region of the EM spectrum $\left(\mathrm{r}_{\mathrm{e}} \sim 10^{-15} \mathrm{~m}\right.$ is large compared to the minimum length of physics). $[33,34]$

The spectral energy density of the Cerenkov radiation in vacuum is by Eq. (86) 


$$
\mathrm{dW} / \mathrm{d} \omega=\mathrm{L}\left(\mu_{\mathrm{o}} \mathrm{e}^{2} / 4 \pi\right)|(\mathrm{v}-\mathrm{w}) / \mathrm{v}|^{3}\left[1-\mathrm{c}_{\mathrm{J}}^{2} /(\mathrm{v}-\mathrm{w})^{2}\right] \omega, \quad 0 \leq \omega \leq \omega_{\mathrm{o}} .
$$

FIG. 3 depicts $d W / d \omega$ versus $0 \leq \omega \leq \omega_{0}$ for various dimensionless charge speeds $B_{v}=$ $|\mathbf{v}| / c_{o}$, one dimensionless ether speed $B_{w}=|\mathbf{w}| / c_{o}=10^{-3}$ (corresponding to its terrestrial value), and antiparallel $v$ and $w$. It is seen that for a given frequency $\omega, d W / d \omega$ is increasing with $B_{v}$.

It is remarkable that spectral radiation energy densities $\mathrm{dW} / \mathrm{d} \omega \propto \omega$ have been observed in interstellar spaces in the presence of cosmic rays (high energy electrons, protons, and charged mesons). [36] This Cerenkov radiation was tentatively interpreted as an ordinary Cerenkov effect in an interstellar space filled with a very dilute plasma with a dielectric permittivity $\epsilon(\omega)>\epsilon_{\mathrm{o}}$. [36]

As known from redshift measurements, distant galaxies move with relativistic velocities $0.1 c_{o}$ $<\mathrm{u}<0.9 \mathrm{c}_{\mathrm{o}}$. [29] The ether in the galactic spaces is probably dragged along by the galaxies through gravitational interaction (expanding universe). The predicted vacuum Cerenkov effect could thus be observable when high-energy cosmic rays travel upstream the galactic ether flow w. The possibility of large ether velocities $w \sim c_{o}$ in the regions of distant galaxies is also suggested by the observed redshift anomaly. [29]

\section{GENERALIZED GALILEI COVARIANT EM FIELD EQUATIONS}

The generalized EM wave and Maxwell equations are derived for arbitrary inertial frames $\Sigma(r, t, w)$ in vacuum with ether flow w, and shown to be Galilei covariant. According to Maxwell, Heaviside, Hertz, Poincare, Lorentz, and Abraham, [2] the usual Maxwell equations hold in the ether rest frame $\Sigma^{\circ}\left(\mathbf{r}^{\circ}, t^{\circ}, 0\right)$. The EM vacuum fields $E^{\circ}\left(\mathbf{r}^{\circ}, t^{\circ}\right)$ and $\mathbf{H}^{\circ}\left(\mathbf{r}^{\circ}, t^{\circ}\right)$ in $\Sigma^{\circ}$ are derivable from vector $\mathbf{A}^{\mathrm{o}}\left(\mathbf{r}^{\mathrm{o}}, \mathrm{t}^{\mathrm{o}}\right)$ and scalar $\Phi^{\mathrm{o}}\left(\mathbf{r}^{\mathrm{o}}, \mathbf{t}^{\mathrm{o}}\right)$ potentials by

$$
\mathbf{E}^{\mathrm{o}}=-\nabla^{\mathrm{o}} \Phi^{\mathrm{o}}-\partial \mathbf{A}^{\mathrm{o}} / \partial \mathrm{t}^{\mathrm{o}}, \mathbf{H}^{\mathrm{o}}=\mu_{\mathrm{o}}^{-1} \nabla^{\mathrm{o}} \times \mathbf{A}^{\mathrm{o}} .
$$

Since Eq. (89) determines only the curl of $\mathbf{A}^{\circ}$, the divergence of $\mathbf{A}^{\circ}$ is subject to the Lorentz gauge,

$$
\nabla^{0} \bullet A^{\circ}=-\mu_{0} \epsilon_{o} \partial \Phi^{\circ} / \partial t^{\circ},
$$

in order to completely specify the vector field $A^{\circ}\left(r^{0}, t^{0}\right)$. By means of Eqs. (88) and (89), the Maxwell equations for the vacuum $\left(\mu_{\mathrm{o}}, \epsilon_{\mathrm{o}}\right)$ can be separated into inhomogeneous wave equations for the EM potentials:

$$
\begin{aligned}
& \mu_{\mathrm{o}} \epsilon_{\mathrm{o}} \partial^{2} \mathbf{A}^{\mathrm{o}} / \partial \mathrm{t}^{\mathrm{o} 2}-\nabla^{\mathrm{o} 2} \mathbf{A}^{\mathrm{o}}=\mu_{\mathrm{o}} \mathbf{j}^{\mathrm{o}}, \\
& \mu_{\mathrm{o}} \epsilon_{\mathrm{o}} \partial^{2} \Phi^{\mathrm{o}} / \partial \mathrm{t}^{\mathrm{o} 2}-\nabla^{\mathrm{o} 2} \Phi^{\mathrm{o}}=\rho^{\mathrm{o}} / \epsilon_{\mathrm{o}} .
\end{aligned}
$$

The EM vacuum potentials have their sources in the space charge $\rho^{\circ}\left(\mathbf{r}^{\circ}, t^{\circ}\right)$ and current $j^{\circ}\left(\mathbf{r}^{\mathrm{o}}, \mathbf{t}^{\mathrm{o}}\right)$ density fields of individual charges e moving with a velocity field $\mathbf{v}^{\circ}\left(\mathbf{r}^{\circ}, t^{\circ}\right)$, where $j^{o}=\rho^{\circ} \mathbf{v}^{o}$.

The EM wave equations (90)-(91) are not generally valid, since they are only applicable in the inertial frame $\Sigma^{\circ}\left(\mathbf{r}^{0}, t^{0}, 0\right)$, in which the wave carrier is at rest, $w^{0}=0$. [3] For this reason, it is physically not meaningful to investigate their covariance properties in space-time transformations (e.g., the Lorentz transformations). [25-26]

Accordingly, the wave equations (90)-(91) are first transformed from the ether rest frame $\Sigma^{\circ}$ to an arbitrary inertial frame $\Sigma(\mathbf{r}, \mathbf{t}, \mathbf{w})$ which moves with an arbitrary constant velocity $\mathbf{U}=-\mathbf{w}$ relative to $\Sigma^{\circ}$. This transformation cannot be accomplished by means of the Lorentz 
transformations, since these do not lead to different wave equations.

Let Eqs. (90)-(91) be transformed by means of a Galilei transformation $\Sigma^{\circ} \rightarrow \Sigma$ to the inertial frame $\Sigma(r, t, w)$ with ether flow $w$. Thus, different wave equations are obtained for the EM vacuum potentials $A(r, t)$ and $\Phi(r, t)$ in the inertial frame $\Sigma(r, t, w)$, that moves with arbitrary constant velocity $\mathbf{U}=-\mathbf{w}$ relative to $\Sigma^{\mathbf{o}}$ :

$$
\begin{gathered}
{\left[\mu_{\mathrm{o}} \epsilon_{\mathrm{o}}(\partial / \partial \mathrm{t}+\mathbf{w} \bullet \nabla)^{2}-\nabla^{2}\right] \mathbf{A}=\mu_{\mathrm{o}}(\mathbf{j}-\rho w)} \\
{\left[\mu_{\mathrm{o}} \epsilon_{\mathrm{o}}(\partial / \partial t+w \bullet \nabla)^{2}-\nabla^{2}\right](\Phi-w \bullet \mathbf{A})=\rho / \epsilon_{\mathrm{o}}}
\end{gathered}
$$

In this Galilei transformation, the vacuum properties have been assumed to be invariant, $\mu=\mu_{\mathrm{o}}$, $\epsilon=\epsilon_{\mathrm{o}}$. Note that the wave equations of the inertial frame $\Sigma(r, t, w)$ contain explicitly the ether velocity $\mathbf{w}$.

From the simultaneous solutions $A(r, t)$ and $\Phi(r, t)$ of Eqs. (92) and (93), the EM fields follow via the covariant relations,

$$
\mathbf{E}=-\nabla \Phi-\partial \mathbf{A} / \partial \mathrm{t}, \quad \mathbf{H}=\mu_{\mathrm{o}}^{-1} \nabla \times \mathbf{A} .
$$

In the inertial frame $\Sigma(r, t, w)$ with ether flow $\mathbf{w}$, the EM potentials satisfy the ether gauge, [25]

$$
\nabla \bullet A=-\mu_{0} \epsilon_{0}(\partial / \partial t+w \bullet \nabla)(\Phi-w \bullet A)
$$

The wave equations of the form (92)-(93) are valid in any inertial frame $\Sigma$, if they are covariant in Galilean space-time transformations from the inertial frame $\Sigma(r, t, w)$ to an arbitrary other inertial frame $\Sigma^{\prime}\left(\mathbf{r}^{\prime}, t^{\prime}, w^{\prime}\right)$ :

$$
\mathbf{r}^{\prime}=\mathbf{r}-\mathbf{u t}, \mathrm{t}^{\prime}=\mathrm{t},
$$

with

$$
\partial / \partial t=\partial / \partial t^{\prime}-\mathbf{u} \bullet \nabla^{\prime}, \nabla=\nabla^{\prime},
$$

where $\Sigma^{\prime}$ moves relative to $\Sigma$ with arbitrary constant velocity $\mathbf{u}\left(0\right.$ of $\Sigma$ and $0^{\prime}$ of $\Sigma^{\prime}$ are assumed

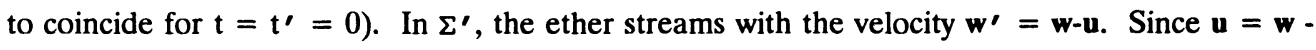
$\mathbf{w}^{\prime}$, Eq. (97) implies the covariant operators,

$$
\partial / \partial \mathrm{t}+\mathrm{w} \bullet \nabla=\partial / \partial \mathrm{t}^{\prime}+w^{\prime} \bullet \nabla^{\prime}, \nabla=\nabla^{\prime} .
$$

In the Galilei transformations $\Sigma \rightarrow \Sigma^{\prime}$, the EM potentials $A, \Phi$ and the source fields $\rho, j$ exhibit the invariance properties, [25]

$$
\begin{gathered}
\mathbf{A}=\mathbf{A}^{\prime}, \rho=\rho^{\prime}, \\
\Phi-\mathbf{w} \bullet \mathbf{A}=\Phi^{\prime}-\mathbf{w}^{\prime} \bullet \mathbf{A}^{\prime}, \\
\mathbf{j}-\rho \mathbf{w}=\mathbf{j}^{\prime}-\boldsymbol{\rho}^{\prime} \mathbf{w}^{\prime} .
\end{gathered}
$$

In view of the Galilei invariants (99)-(101), and the covariant operators in Eq. (98), it is easily recognized that the wave equations are of the same form for $A^{\prime}\left(r^{\prime}, t^{\prime}\right)$ and $\Phi^{\prime}\left(r^{\prime}, t^{\prime}\right)$ of $\Sigma^{\prime}$ as the wave equations (92) and (93) of $\Sigma$, if the vacuum properties are invariant,

$$
\mu_{0}=\mu_{0}^{\prime}, \epsilon_{0}=\epsilon_{0}^{\prime} .
$$

Accordingly, the wave equations (92) and (93) are Galilei covariant, i.e., they are applicable 
in any arbitrary inertial frame $\Sigma(r, t, w)$ with ether flow $w$. The invariants (98)-(102) with $\Sigma^{\prime} \equiv \Sigma^{0}$, $w^{\prime} \equiv w^{0}=0$, can also be used to transform the wave equations (92)-(93) into the wave equations (91)-(92) of the ether frame $\Sigma^{\circ}$.

Since $\mathbf{u}=\mathbf{w}-\mathbf{w}^{\prime}=\mathbf{v}-\mathbf{v}^{\prime}$ in the Galilei transformations $\Sigma \rightarrow \Sigma^{\prime}$, Eqs. (98), (99), and (100) imply the Galilei invariants,

$$
\begin{gathered}
\partial \partial \mathrm{t}+\mathbf{v} \bullet \nabla=\partial / \partial \mathrm{t}^{\prime}+\mathbf{v}^{\prime} \bullet \nabla^{\prime}, \\
\Phi-\mathbf{v} \bullet \mathbf{A}=\Phi^{\prime}-\mathbf{v}^{\prime} \bullet \mathbf{A}^{\prime}, \\
\mathbf{j}-\rho \mathbf{v}=\mathbf{j}^{\prime}-\rho^{\prime} \mathbf{v}^{\prime} .
\end{gathered}
$$

Application of Eq. (94) to the Galilei invariants (99), (100), and (104) yields, under consideration of Eq. (102), the EM field invariants in Galilei transformations $\Sigma \rightarrow \Sigma^{\prime}$ :

$$
\begin{gathered}
\mathbf{E}+\mu_{\mathrm{o}} \mathbf{w} \times \mathbf{H}=\mathbf{E}^{\prime}+\mu_{\mathrm{o}} \mathbf{w}^{\prime} \times \mathbf{H}^{\prime}, \\
\mathbf{E}+\mu_{\mathrm{o}} \mathbf{v} \times \mathbf{H}=\mathbf{E}^{\prime}+\mu_{\mathrm{o}} \mathbf{v}^{\prime} \times \mathbf{H}^{\prime}, \\
\mathbf{H}=\mathbf{H}^{\prime},
\end{gathered}
$$

where

$$
\mathbf{w}-\mathbf{w}^{\prime}=\mathbf{v}-\mathbf{v}^{\prime}=\mathbf{u} .
$$

The corresponding Galilei covariant generalized Maxwell equations for the EM vacuum $\left(\mu_{\mathrm{o}}\right.$, $\epsilon_{\mathrm{o}}$ ) fields $\mathbf{E}(\mathbf{r}, \mathrm{t}), \mathbf{H}(\mathbf{r}, \mathbf{t})$ in an arbitrary inertial frame $\Sigma(\mathbf{r}, t, \mathbf{w})$ with ether flow $\mathbf{w}$ are given by: [25]

$$
\begin{gathered}
\nabla \times\left(\mathbf{E}+\mu_{\mathrm{o}} \mathbf{w} \times \mathbf{H}\right)=-(\partial / \partial \mathrm{t}+\mathbf{w} \bullet \nabla) \mu_{\mathrm{o}} \mathbf{H}, \\
\nabla \bullet \epsilon_{\mathrm{o}}\left(\mathbf{E}+\mu_{\mathrm{o}} \mathbf{w} \times \mathbf{H}\right)=\boldsymbol{\rho}, \\
\nabla \times \mathbf{H}=(\partial / \partial \mathrm{t}+\mathbf{w} \bullet \nabla) \epsilon_{\mathrm{o}}\left(\mathbf{E}+\mu_{\mathrm{o}} \mathbf{w} \times \mathbf{H}\right)+\mathbf{j}-\rho \mathbf{w}, \\
\nabla \bullet \mu_{\mathrm{o}} \mathbf{H}=0 .
\end{gathered}
$$

The Galilei covariance of Eqs. (110)-(113) follows from the Galilei invariants (98), (101), (102), (106), and (108). For $\mathbf{w}=\mathbf{0}$, the generalized Maxwell equations (110)-(113) reduce to the ordinary Maxwell equations, which hold only in the ether frame $\Sigma^{\circ}$. Similarly, application of the invariants (98), (101), (102), (106), and (108) to the Galilei transformation $\Sigma \rightarrow \Sigma^{\circ}\left(w^{\prime}=w^{0} \equiv 0\right)$ transforms Eqs. (110)-(113) to the Maxwell equations of the ether frame $\Sigma^{\circ}$.

As to a more detailed derivation of the Galilei covariant EM field equations for inertial frames with ether flow, it is referred to the original publication for dielectric media, which also contains several other applications. [25]

\section{CONCLUSION}

In applied science and engineering, the Galilean transformations for the EM field have found widespread use (e.g., in the theory of EM induction generators and motors). On the other hand, the STR is frequently avoided because of the physical contradictions it generates in non-trivial applications (see, e.g. the contradictions associated with the nonlinear velocity addition theorem). [37] Furthermore, the Lorentz covariant Maxwell equations have been shown to have an infinite 
number of linear and nonlinear covariant transformations (non-uniqueness of Lorentz transformations). [38] It has been the merit of Builder, who demonstrated that the EM forces between moving charges and magnetic dipoles do not depend on their relative velocities (as postulated by the STR), but on their absolute velocities. [10,11] Thus, Builder clearly proved that the Galilean relativity principle holds and that of the STR fails.

In order to test the presented results, and the Galilean relativity principle in general, it is necessary to carry through specific experiments and to interpret the experimental data by means of the Galilei covariant EM equations. Winterberg proposed experiments to verify the breakdown of the STR kinematics due to the non-zero relaxation time for (i) length contraction of bodies set into absolute motion in the ether and (ii) time dilation of clocks consisting of an EM signal reflected hence and force between mirrors held apart by a rod. [15,17] The following experiments appear to be promising for the further investigation of the substratum physics of the vacuum.

Intense high-energy electron beams travelling upstream the terrestrial ether flow should be used to excite Cerenkov radiation in vacuum. Since the spectral radiation intensity $d W / d \omega \propto \omega$ is proportional to the frequency $0<\omega<\omega_{0}$, radiation detectors sensitive at high frequencies are required. Furthermore, it might be possible to observe vacuum Cerenkov radiation generated by cosmic rays with sensitive probes in regions of interstellar space with large ether velocities $w \sim c_{0}$.

Sagnac type experiments have already proven the effect of ether flow on light propagation. [2] Similarly, one could probably employ high-energy electron beams to verify the anisotropy of space due to the local ether flow w. In particular, one could study the slowing down of a superluminal electron beam $\left(|\mathbf{v}-\mathbf{w}|>\mathrm{c}_{\mathrm{o}}\right)$ moving upstream the terrestrial ether flow $w$ due to the EM wave drag force per electron, [27]

$$
f=-[(v-w) /|v-w|][(v-w) / v]^{2}\left(e^{2} / 3 \pi \epsilon_{o} r_{e}^{2}\right)\left[1-c_{j}^{2} /(v-w)^{2}\right], \quad|v-w|>c_{o} .
$$

An electron beam of the same type directed downstream the ether flow w would have different slowing down characteristics.

It is now widely recognized that the STR is a tautology which gives approximately correct answers in applications where $|w|<<c_{o}$ and completely false results when $|w| \sim c_{0} \cdot[2,4-20,30-36]$ However, considerable theoretical and experimental research remains to be done to get a more comprehensive picture of Galilei covariant theories at high energies.

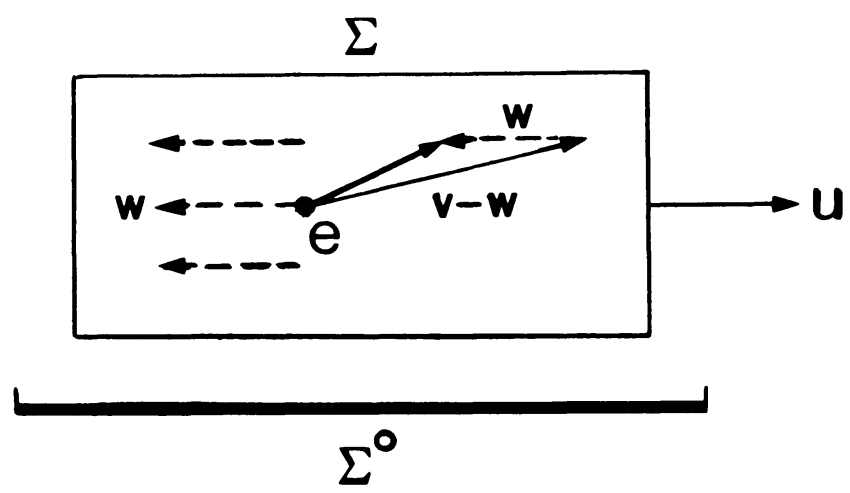

FIG. 1: Charge e moving with velocity $\mathbf{v}$ in inertial frame $\Sigma$ with substratum flow $\mathbf{w}$. 


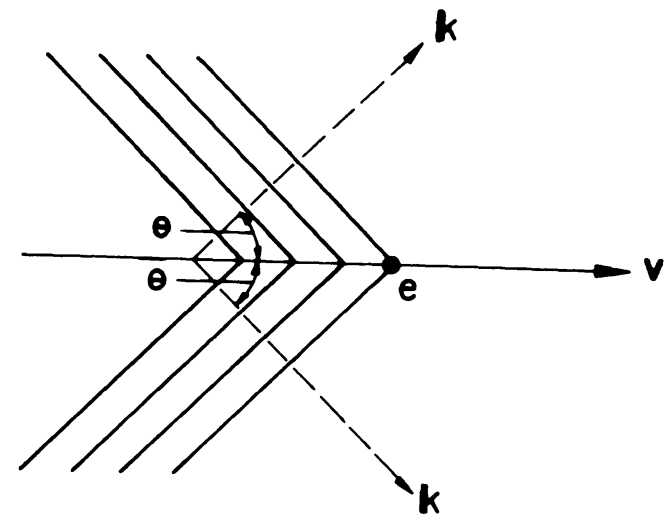

FIG. 2: Wave fronts of EM shock wave in substratum, with moving charge e at vertex of Mach cone.

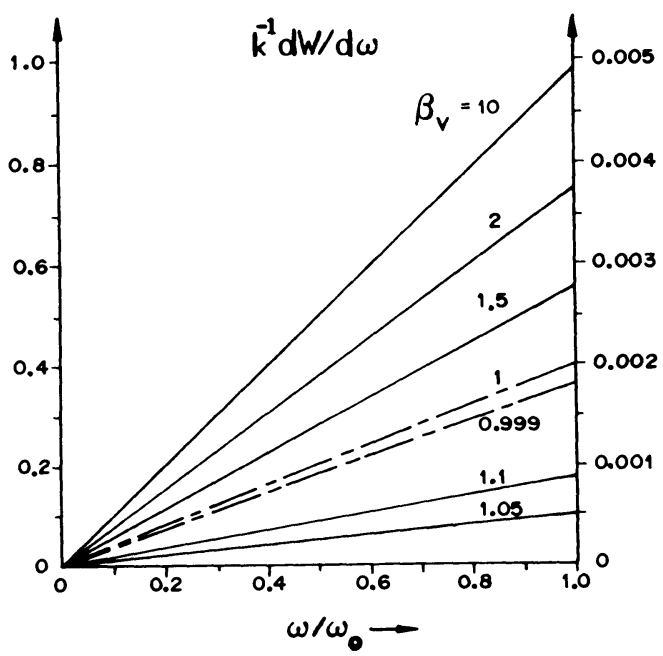

FIG. 3: Spectral radiation density $d W / d \omega$ versus $\omega / \omega_{o}$ for $B_{w}=10^{-3}$ and various $B_{v}$ $\left(\mathrm{K}=\mu_{\mathrm{o}} \mathrm{Le}^{2} \omega_{\mathrm{o}} / 4 \pi\right)$.

\section{REFERENCES}

1. SOMMERFELD, A. Dynamics of Uniformly Moving Charge, Goett. Nachr. 99, (1904), 363368.

2. JANOSSY, L. Relativity Based on Physical Reality, Akademiai Kiado, Budapest, 1972.

3. GINZBURG, V.L. Certain Theoretical Aspects of Radiation Due to Superluminal Motion in a Medium. Sov. Phys. Uspekhi 2, (1960), 874-893.

4. IVES, H.E. The Fitzgerald Contraction, Proc. R. Dublin Soc. 26, (1952), 9-26.

5. IVES, H.E. Genesis of the Query "Is there and Ether?", J. Opt. Soc. Am. 43, (1952), 217-218.

6. JANOSSY, L. Physical Interpretation of Lorentz Transformation, Ann Physik. 11, (1953), 293 322.

7. JANOSSY, L. Reflections on the Problem of Measuring the Velocity of Light, Acta Phys. Hung. 17, (1963), 421-455.

8. DIRAC, P.A.M. Is There an Ether?, Nature 168, (1951), 906-1908.

9. DIRAC, P.A.M. The Principles of Quantum Mechanics, Clarendon, Oxford, 1958.

10. BUILDER, G. Ether and Relativity, Aust. J. Phys. 11, (1958), 279-297.

11. BUILDER, G. The Constancy of the Velocity of Light, Aust. J. Phys. 11, (1958), 457-480.

12. MANSOURI, R. and SEXL, R.U. A Test of Special Theory of Relativity: Simultaneity and Clock Synchronization, Gen. Rel. Grav. ㅁ, (1977), 497-513. 
13. WINTERBERG, F. Nonlinear Generalization of Relativity at Very High Energies, Atomkernenergie 44, (1984), 238-246.

14. WINTERBERG, F. Dynamic Effects Violating Relativity, Z. Naturforsch. 41a, (1986), 12611266.

15. WINTERBERG, F. Possible Evidence for Weak Violation of Special Relativity, $\underline{Z}$. Naturforsch. 42a, (1987), 1374-1375.

16. WINTERBERG, F. Lorentz Invariance as a Dynamic Symmetry, Z. Naturforsch. 42a, (1987), 1428-1442.

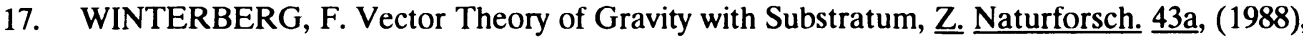
369-384.

18. WINTERBERG, F. Substratum Approach to a Unified Theory of Elementary Particles, $\underline{\mathbf{Z}}$. Naturforsch, 43a, (1988), 1131-1150.

19. EINSTEIN, A.: Geometry and Experience, Preuss. Akad. Wissensch., (1921), 123-128: "According to the general theory of relativity space without ether is unthinkable; for in such space there not only would be no propagation of light, but also no possibility of existence for standards of space and time (measuring rods and clocks), nor therefore any space-time in a physical sense."

20. BRILLOUIN, L. Relativity Reexamined, Academic, New York, 1970.

21. HENRY, P.S. Isotropy of 3K Background, Nature 231, (1971), 516-519.

22. PENZIAS, A.A. and WILSON, R.W. Measurement of Excess Antenna Temperature at 4080 Mc/s, Ap. J. 142, (1965), 419-421.

23. FRANKL, F.I. and KARPOVICH, E.A. Gas Dynamics of Thin Bodies, Interscience, New York, 1953.

24. WILHELM, H.E. Galilei Covariant Electromagnetic Field Equations, Bull. Am. Phys. Soc. $\underline{29},(1984), 433 ; \underline{29},(1984), 732$.

25. WILHELM, H.E. Covariant Electromagnetic Theory for Inertial Frames with Substratum Flow, Radio Science 20, (1985), 1006-1018.

26. WILHELM, H.E. Galilei Covariant Quantum Mechanics in Electromagnetic Fields, $\underline{\text { Int. }} \underline{\mathbf{J}}$. Math. $\underline{\text { Math. }}$ Sci. $\underline{8}$, (1983), 589-597.

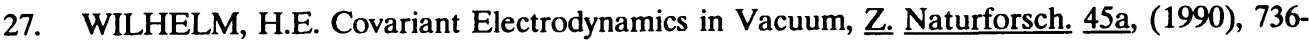
748.

28. WILHELM, H.E. Lorentz Transformation as a Galilei Transformation with Physical Length and Time Contractions, $\underline{Z}$. Naturforsch. $\underline{43 a}$, (1988), 859-864.

29. HUMASON, M.L. Red Shifts in the Spectra of Extragalactic Nebulae, Vistas in Astronomy 2, 1620-1628, (Ed. A. Beer) Pergamon, New York, 1956.

30. GRANDY, W.T., JR. Introduction to Electrodynamics and Radiation, Academic, New York, 1970.

31. GRANDY, W.T., JR. and AGHAZADEH, A. Radiative Corrections for Extended Charged Particles in Classical Electrodynamics, Ann. Phys. 142, (1982), 284-298.

32. DIRAC, P.A.M. On the Existence of the Ether, Europhys. News $\underline{8}$, (1977), 1-3.

33. HEISENBERG, W. The Universal Length in the Theory of Elementary Particles, $\underline{\text { Ann. Physik }}$ 32, (1938), 20-33.

34. WHEELER, J.A. Geometrodynamics, Academic, New York, 1962.

35. ALFVEN, H. Cosmology: Myth or Science, Cosmology, (Eds. W. Yourgrau and A.D. Breck) 1-13, Plenum, New York, 1977.

36. GINZBURG, V.L. Theoretical Physics and Astrophysics, Pergamon, New York, 1979.

37. MECANU, C.I. Some Difficulties within the Framework of Relativistic Electrodynamics, Archiv Elektrotech. 69, (1986), 97-108.

38. WILHELM, H.E. Transformation Method for Electromagnetic Wave Problems with Moving Boundary Conditions, Archiv Elektrotech. 72, (1989), 165-173. 


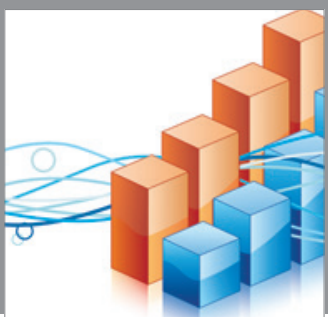

Advances in

Operations Research

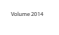

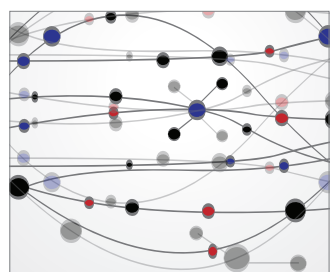

\section{The Scientific} World Journal
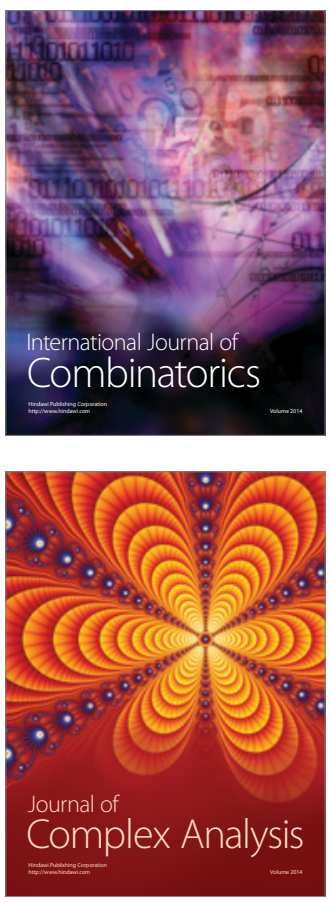

International Journal of

Mathematics and

Mathematical

Sciences
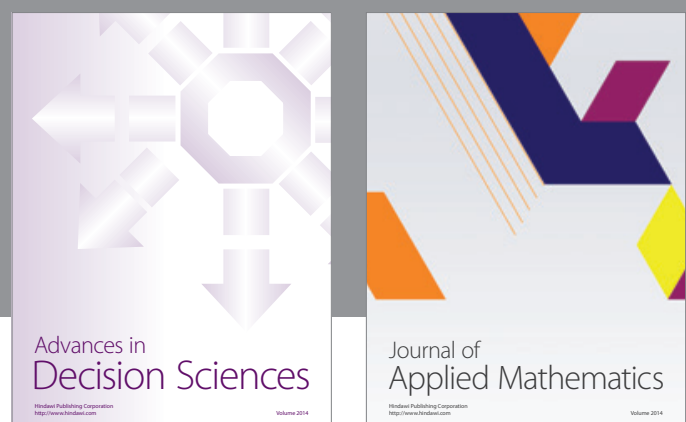

Journal of

Applied Mathematics
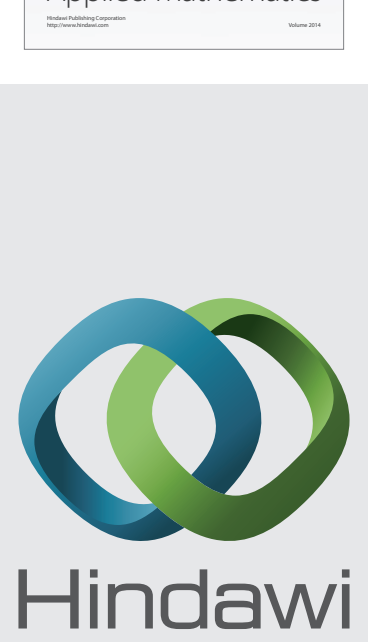

Submit your manuscripts at http://www.hindawi.com
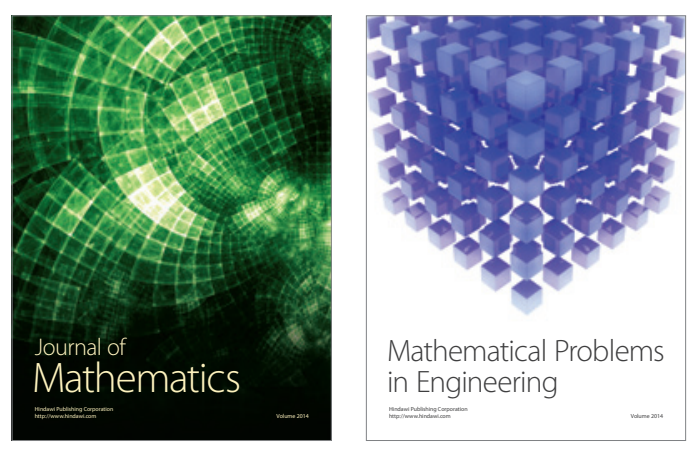

Mathematical Problems in Engineering
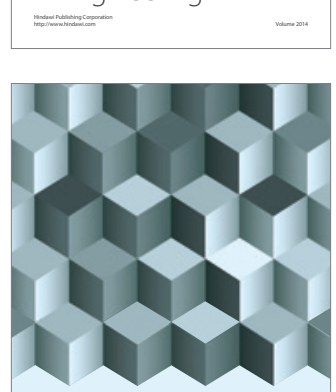

Journal of

Function Spaces
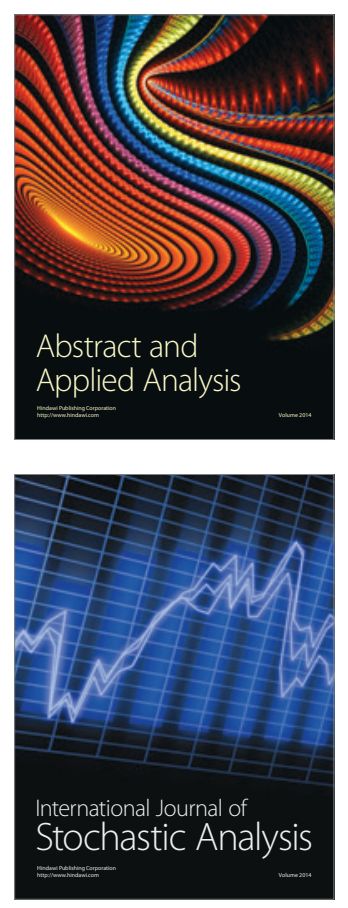

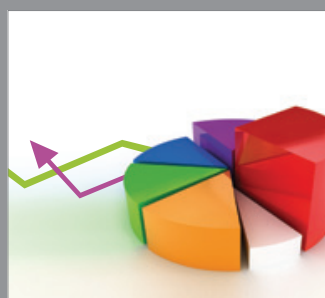

ournal of

Probability and Statistics

Promensencen
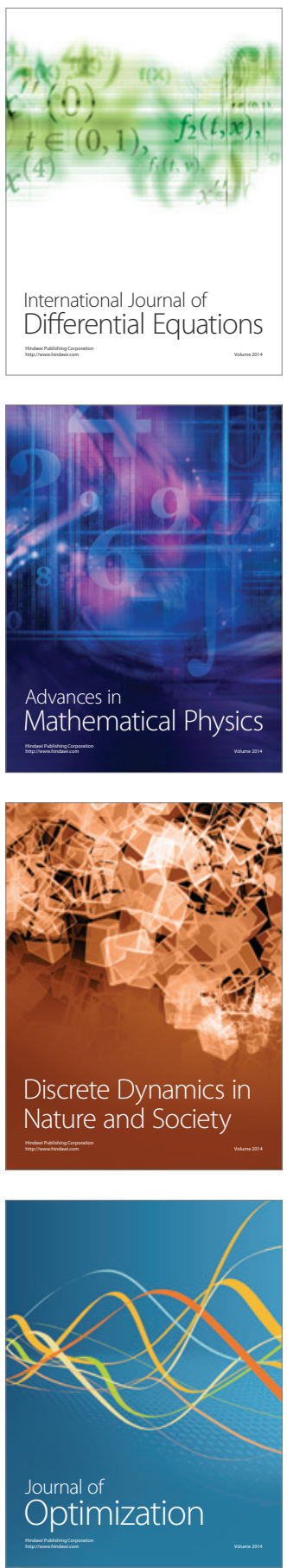